TRANSACTIONS OF THE

AMERICAN MATHEMATICAL SOCIETY

Volume 351, Number 9, Pages 3771-3792

S 0002-9947(99)02101-7

Article electronically published on March 1, 1999

\title{
CAUSAL COMPACTIFICATION AND HARDY SPACES
}

\author{
G. ÓLAFSSON AND B. ØRSTED
}

\begin{abstract}
Let $\mathcal{M}=G / H$ be a irreducible symmetric space of Cayley type. Then $\mathcal{M}$ is diffeomorphic to an open and dense $G$-orbit in the Shilov boundary of $G / K \times G / K$. This compactification of $\mathcal{M}$ is causal and can be used to give answers to questions in harmonic analysis on $\mathcal{M}$. In particular we relate the Hardy space of $\mathcal{M}$ to the classical Hardy space on the bounded symmetric domain $G / K \times G / K$. This gives a new formula for the Cauchy-Szegö kernel for $\mathcal{M}$.
\end{abstract}

\section{INTRODUCTION}

In this paper we shall begin a study of the harmonic analysis of a certain class of affine symmetric spaces by introducing a compactification of the space. We shall call these causal compactifications. The idea of using such compactifications has been studied before in special cases, see for example [1], [9] and [10], where Cayley type spaces are treated as well as the case of the metaplectic group, and also recently by V. Molchanov for the case of the orthogonal group of Hermitian type and the corresponding hyperboloid [12]. The causal compactification is especially convenient for studying the noncommutative analogue of the Hardy space, since it is a Shilov boundary of a classical bounded domain. Such non-classical Hardy spaces were introduced by Stanton in [20] in the group case, also studied by Ol'shanskii, and later constructed for symmetric spaces of Hermitian type in [6].

One may trace some of these ideas to the celebrated Gelfand-Gindikin program of understanding the harmonic analysis on a symmetric space by studying the holomorphic extension of functions into domains in the complexification of the space. One of the first questions was to calculate the reproducing kernel for the Hardy space, in the sense of finding the orthogonal projection from $L^{2}$ onto the Hardy space, i.e. to find explicitly the Cauchy-Szegö kernel which reproduces the holomorphic functions in the Hardy space.

One of our aims in this paper is, in addition to the harmonic analysis treated, to make precise the notion of causal compactification. For symmetric spaces of Cayley type there is, as we shall see in this paper, a natural way to obtain a causal compactification and to use it to identify the Hardy space and its Cauchy-Szegö kernel explicitly in terms of classical Hardy spaces, namely a tensor product of two such spaces corresponding to the associated tube domain. By specializing our results we obtain as a corollary an explicit formula for the Cauchy-Szegö kernel

Received by the editors April 29, 1996 and, in revised form, March 10, 1997.

1991 Mathematics Subject Classification. Primary 43A85, 22E46; Secondary 43A65, 53C35.

Key words and phrases. Causal compactification, Hardy spaces, holomorphic discrete series.

The first named author was supported by NSF grant DMS-9626541, LEQSF grant (1996-99)RD-A-12 and the Danish Research Council. 
for the two-dimensional hyperboloid of one sheet - namely a special case of an old formula of Heine:

$$
\frac{1}{t-z}=\sum_{n=0}^{\infty}(2 n+1) P_{n}(z) Q_{n}(t),
$$

see e.g. [21] p. 322. (We thank J. Faraut for this observation.) Here the functions in the sum are Legendre functions of the first and second kind. Our formula is obtained by setting $z=1$ and is the expression of the Cauchy-Szegö kernel as a sum of the invariant distributions which define the projections onto the holomorphic discrete series representations for the symmetric space. The numerical coefficients in the sum are due to the standard normalization of the Legendre functions, but as shown in [6] the multiplicity is one in general, so in principle the coefficients are all 1.

Although in this paper we deal only with affine symmetric spaces of Cayley type, it is plausible that the ideas and constructions carry over to quite general (reductive) affine symmetric spaces $G / H$ of Hermitian type. Namely, for such a (general) space there will be a causal compactification into the Shilov boundary of a Hermitian symmetric space $G_{1} / K_{1}$, where $G_{1}$ is the group of local causal transformations on $G / H$. This compactification map extends to a holomorphic imbedding of $\Xi$, the domain in a suitable complexification of $G / H$ for a Hardy space of functions on $G / H$. In this way it is possible to directly compare the Hardy space on $G / H$ with the classical one on the Shilov boundary of $G_{1} / K_{1}$; to have an isomorphism between the two Hardy spaces will require some control over the asymptotics at infinity in $\Xi$ of the functions in the Hardy space. This is an interesting program, and several researchers are presently studying special cases; we hope to pursue it with regard to calculating the Cauchy-Szegö kernels, and also to utilize the compactifications to study the other series of representations as in the original program of Gelfand and Gindikin.

The main result in this paper is Theorem 6.6, complemented by Theorem 6.9, giving an explicit isomorphism between the classical Hardy space and the one for the symmetric space. Note that in this case the causal group is $G_{1}=G \times G$, so that the corresponding Hardy space is actually a tensor product of the Hardy space on $G / K$ tensor itself. Hence we may interpret the result as calculating this tensor product and identifying it with the direct sum of all holomorphic discrete series representations for $G / H$ (it is also possible to do this tensor product calculation directly by algebraic means - this we did first - and to compare it with the parameters in the holomorphic discrete series for $G / H)$. We start by recalling the structure theory of Cayley type spaces where a number of new technical aspects have to be dealt with. One is the normalization of various measures and corresponding Jacobians, as we do in Sections 2 and 5. Another is the introduction of a double covering, necessary in two of the series of spaces, similar to the one appearing in [10]. It seems a natural feature of the geometry that one should consider a double covering of $\Xi$, defined much in the spirit of the Riemann surface for $\sqrt{z}$, but with $z$ replaced by the complex Jacobian of the (extended) causal compactification.

It is a pleasure to thank P. Torasso for the invitation to Poitiers, where we first lectured on these results, J. Faraut and K. Koufany for remarks and discussions, and Odense University and the Mittag-Leffler Institute, where this work was finished. We also thank the referee and the communicating editor for many suggestions to improve the presentation. 


\section{Symmetric Spaces of Cayley Type}

In this section we recall some facts about causal symmetric spaces. We refer to the original paper [14] and the monograph [5] for proofs and more information. Let $\mathbf{V}$ be a Euclidean space. A subset $C$ in $\mathbf{V}$ is called a cone if $C$ is convex and $\mathbb{R}^{+} C \subset C$. The closed cone $C$ is called nontrivial if $C \neq-C$ and regular if it is pointed $(C \cap-C=\{0\})$ and generating $(C-C=\mathbf{V})$. Suppose that the Lie group $L$ acts on $\mathbf{V}$. We say that the cone $C$ is $L$-invariant if $a(C) \subset C$ for all $a \in L$. Denote by $\operatorname{Cone}_{L}(\mathbf{V})$ the set of regular $L$-invariant cones in $\mathbf{V}$. Let $\mathcal{M}$ be a manifold. A cone field or causal structure on $\mathcal{M}$ is a map

$$
C: \mathcal{M} \ni m \mapsto C(m) \subset T_{m}(\mathcal{M}),
$$

where $C(m)$ is a closed nontrivial cone in $T_{m}(\mathcal{M}), m \in \mathcal{M}$. The cone field is said to be differentiable if there exists a differentiable atlas $A$ on $T(M)$ such that for $(\varphi, U) \in A$ there exits an open $V \subset \mathbb{R}^{m}$ and a closed cone $C \subset \mathbb{R}^{m}, m=\operatorname{dim} \mathcal{M}$, such that $\operatorname{pr}_{2}(\varphi(C(m)))=C, m \in U$. Here $\operatorname{pr}_{2}: \mathbf{V} \times \mathbb{R}^{m} \rightarrow \mathbb{R}^{m}$ is the projection onto the second factor. Suppose that the Lie group $G$ acts on $\mathcal{M}$. Let $\ell_{g}(m):=g \cdot m$. The cone field is said to be invariant if $\left(d \ell_{g}\right)(C(m))=C(g \cdot m) \subset T_{g \cdot m}(\mathcal{M})$. If the action is transitive, then $\mathcal{M}$ is said to be causal if there exists a smooth $G$-invariant cone field on $\mathcal{M}$. If $\mathcal{M}$ is causal, then $C(m)$ is invariant under $G^{m}=\{g \in G \mid$ $g \cdot m=m\}$. On the other hand if $C \subset T_{m}(\mathcal{M})$ is a $G^{m}$ invariant cone, then the assignment $g \cdot m \mapsto\left(d \ell_{g}\right)_{m}(C)$ defines an invariant causal structure on $\mathcal{M}$. Hence the $G$-invariant causal structures on $\mathcal{M}$ correspond bijectively to the $G^{m}$-invariant cones in $T_{m}(\mathcal{M})$.

Definition 1.1. Let $\mathcal{M}$ and $\mathcal{N}$ be manifolds with causal structure $\mathcal{M} \ni m \mapsto C(m)$ respectively $\mathcal{N} \ni n \mapsto D(n)$. Let $\Phi: \mathcal{M} \rightarrow \mathcal{N}$ be smooth. The pair $(\mathcal{N}, \Phi)$ is called a causal compactification of $\mathcal{M}$ if $\Phi$ is a diffeomorphism onto an open dense subset of $\mathcal{N}$, and $(d \Phi)_{m}(C(m))=D(\Phi(m))$ for all $m \in \mathcal{M}$. Let $G$ be a Lie group acting on $\mathcal{M}$ such that $C$ is $G$-invariant. The causal compactification $(\mathcal{M}, \Phi)$ is called $G$-equivariant if $\mathcal{N}$ is a $G$-space, $\Phi$ is $G$-equivariant, and $D$ is $G$-invariant.

Let $G$ be a connected simple Lie group and $\mathfrak{g}$ be its Lie algebra. Let $G_{\mathbb{C}}$ be the connected simply connected complex Lie group with Lie algebra $\mathfrak{g}_{\mathbb{C}}:=\mathfrak{g} \otimes_{\mathbb{R}} \mathbb{C}$. For simplicity we will assume that $G \subset G_{\mathbb{C}}$. Let $\tau$ be a nontrivial involution on $G$ commuting with a (fixed) Cartan involution $\theta$. We assume that $\tau \neq \theta$. We denote by the same letters the corresponding involutions on $G_{\mathbb{C}}, \mathfrak{g}, \mathfrak{g}_{\mathbb{C}}, \mathfrak{g}^{*}$, and $\mathfrak{g}_{\mathbb{C}}^{*}$. Let $H:=G^{\tau} \subset H_{\mathbb{C}}:=G_{\mathbb{C}}^{\tau}$, and $K:=G^{\theta} \subset K_{\mathbb{C}}:=G_{\mathbb{C}}^{\theta}$. Both $H_{\mathbb{C}}$ and $K_{\mathbb{C}}$ are connected as $G_{\mathbb{C}}$ is simply connected, cf. [11], p. 171 . $K$ is a connected maximal compact subgroup of $G$ and $\mathcal{D}:=G / K$ is a Riemannian symmetric space. Let $\mathcal{M}:=G / H$. $\mathcal{M}$ is a pseudo-Riemannian symmetric space.

For a vector space $\mathbf{V}$ over the field $\mathbb{K}, T \in \operatorname{End}(\mathbf{V})$ and $\lambda \in \mathbb{K}$ let $\mathbf{V}(\lambda, T)=$ $\{v \in \mathbf{V} \mid T(v)=\lambda v\}$. Let $\mathfrak{h}:=\mathfrak{g}^{\tau}=\mathfrak{g}(1, \tau)$ and $\mathfrak{k}:=\mathfrak{g}(1, \theta)$. Then $\mathfrak{h}$ is the Lie algebra of $H$ and $\mathfrak{k}$ is the Lie algebra of $K$. Finally we define $\mathfrak{q}:=\mathfrak{g}(-1, \tau)$ and $\mathfrak{p}:=\mathfrak{g}(-1, \theta)$. Then, as $\tau$ and $\theta$ commute,

$$
\mathfrak{g}=\mathfrak{h} \oplus \mathfrak{q}=\mathfrak{k} \oplus \mathfrak{p}=\mathfrak{h} \cap \mathfrak{k} \oplus \mathfrak{q} \cap \mathfrak{k} \oplus \mathfrak{h} \cap \mathfrak{p} \oplus \mathfrak{q} \cap \mathfrak{p} .
$$

Let $\mathbf{o}=\{H\}$. Then $\mathfrak{q}$ is isomorphic to $T_{\mathbf{o}}(\mathcal{M})$ in such a way that $\left(d \ell_{h}\right)_{\mathbf{o}}$ corresponds to $\operatorname{Ad}(h) \mid \mathfrak{q}, h \in H$. Thus the causal structures on $\mathcal{M}$ correspond bijectively to the $H$-invariant nontrivial cones in $\mathfrak{q}$. There are three different types of causal structures on $\mathcal{M}$ : 
(CC) Compactly causal: there exists a $C \in \operatorname{Cone}_{H}(\mathfrak{q})$ such that $C^{o} \cap \mathfrak{k} \neq \emptyset$.

(NCC) Noncompactly causal: there exists a $C \in \mathrm{Cone}_{H}(\mathfrak{q})$ such that $C^{o} \cap \mathfrak{p} \neq \emptyset$.

(CT) Cayley type: $\mathcal{M}$ is both compactly causal and noncompactly causal.

We say that $\mathcal{M}$ is irreducible if there are no $\tau$-stable ideals in $\mathfrak{g}$ that are not contained in $\mathfrak{h}$. From now on we assume that $\mathcal{M}$ is irreducible. We will also assume that $[\mathfrak{q}, \mathfrak{q}]=\mathfrak{h}$. This is no loss of generality because $[\mathfrak{q}, \mathfrak{q}] \oplus \mathfrak{q}$ is an ideal in $\mathfrak{g}$ and there exists an ideal $\mathfrak{l}$ contained in $\mathfrak{h}$ such that $\mathfrak{g}=\mathfrak{l} \oplus([\mathfrak{q}, \mathfrak{q}] \oplus \mathfrak{q})$. Let $\mathfrak{h}^{a}:=\mathfrak{k} \cap \mathfrak{h} \oplus \mathfrak{p} \cap \mathfrak{q}=\mathfrak{g}^{\tau \theta}$. Denote the center of $\mathfrak{h}^{a}$ by $\mathfrak{z}\left(\mathfrak{h}^{a}\right)$. Denote the center of $\mathfrak{k}$ by $\mathfrak{z}(\mathfrak{k})$. We have, cf. [5, 14]:

Theorem 1.2. Let $\mathfrak{q}^{H \cap K}=\{X \in \mathfrak{q} \mid \forall k \in K \cap H: A d(k) X=X\}$.

1) $\mathcal{M}$ is causal if and only if $\mathfrak{q}^{H \cap K} \neq\{0\}$.

2) $\mathfrak{q}^{H \cap K}=\left(\mathfrak{q}^{H \cap K} \cap \mathfrak{k}\right) \oplus\left(\mathfrak{q}^{H \cap K} \cap \mathfrak{p}\right)$ and $\operatorname{dim} \mathfrak{q}^{H \cap K} \leq 2$.

3) $\operatorname{dim} \mathfrak{q}^{H \cap K}=2$ if and only if $\mathcal{M}$ is of Cayley type.

4) The following statements are equivalent:

i) $\mathcal{M}$ is compactly causal.

ii) $\mathfrak{q}^{H \cap K} \cap \mathfrak{k} \neq\{0\}$.

iii) $\mathfrak{q} \mathfrak{z}(\mathfrak{k}) \neq\{0\}$.

5) The following statements are equivalent:

i) $\mathcal{M}$ is noncompactly causal.

ii) $\mathfrak{q}^{H \cap K} \cap \mathfrak{p} \neq\{0\}$.

iii) $\mathfrak{q} \cap \mathfrak{p} \cap \mathfrak{z}\left(\mathfrak{h}^{a}\right) \neq\{0\}$.

Assume that $\mathcal{M}$ is compactly causal. Then (4) part (iii) implies that $\mathcal{D}$ is a bounded symmetric domain and that we can choose $Z^{0} \in \mathfrak{q} \cap \mathfrak{z}(\mathfrak{k})$ such that ad $Z^{0}$ has exactly the eigenvalues $0,+i,-i$. We have $\mathfrak{k}=\mathfrak{g}\left(0\right.$, ad $\left.Z^{0}\right)$ and $\mathfrak{p}_{\mathbb{C}}$ decomposes

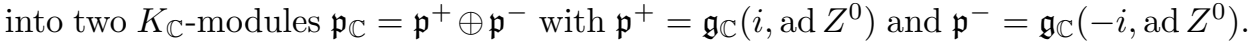
Both $\mathfrak{p}^{+}$and $\mathfrak{p}^{-}$are abelian. The Cartan involution is an inner automorphism given on the Lie algebra by $\theta=\operatorname{Ad}\left(\exp \frac{\pi}{2} Z^{0}\right)=e^{\frac{\pi}{2} \text { ad } Z^{0}}$ and on the group by $\theta=\operatorname{Int}\left(\exp \left([\pi / 2] Z^{0}\right)\right)$, where $\operatorname{Int}(a)$ stands for the inner automorphism $x \mapsto a x a^{-1}$.

Let $P^{+}=\exp \mathfrak{p}^{+}$and $P^{-}=\exp \mathfrak{p}^{-}$. Let $\log :=\left(\exp \mid \mathfrak{p}^{+}\right)^{-1}: P^{+} \rightarrow \mathfrak{p}^{+}$. $P^{+} K_{\mathbb{C}} P^{-}$is open and dense in $G_{\mathbb{C}}$ and $P^{+} \times K_{\mathbb{C}} \times P^{-} \ni(p, k, q) \mapsto p k q \in$ $P^{+} K_{\mathbb{C}} P^{-} \subset G_{\mathbb{C}}$ is a diffeomorphism. Write $g=p(g) k(g) q(g)$ for $g \in P^{+} K_{\mathbb{C}} P^{-}$. Define $\zeta: P^{+} K_{\mathbb{C}} P^{-} \rightarrow \mathfrak{p}^{+}$by $\zeta(g):=\log (p(g))$. The bounded realization of $G / K$, cf. [2] or [3], is given by

$$
\mathcal{D}_{b}=\{\zeta(g) \mid g \in G\} .
$$

For $g \in G_{\mathbb{C}}$ and $Z \in \mathfrak{p}^{+}$with $g \exp Z \in P^{+} K_{\mathbb{C}} P^{-}$, define $g \cdot Z \in \mathfrak{p}^{+}$and $k(g, Z) \in K_{\mathbb{C}}$ by

$$
g \exp Z \in \exp (g \cdot Z) k(g, Z) P^{-} .
$$

The universal factor of automorphy $k(g, Z)$ satisfies

$$
k(a b, Z)=k(a, b \cdot Z) k(b, Z) .
$$

If $\mathcal{M}$ is noncompactly causal, then there is $Y^{0} \in \mathfrak{q} \cap \mathfrak{p}$ such that $\mathfrak{g}=\mathfrak{g}\left(0, \operatorname{ad} Y^{0}\right) \oplus$ $\mathfrak{g}\left(1, \operatorname{ad} Y^{0}\right) \oplus \mathfrak{g}\left(-1, \operatorname{ad} Y^{0}\right)$ and

$$
\mathfrak{h}^{a}=\mathfrak{z}\left(Y^{0}\right):=\mathfrak{g}\left(0, \operatorname{ad} Y^{0}\right) .
$$

Denote the Killing form on $\mathfrak{g}$ by $B$. Set $(X \mid Y)_{\theta}:=-B(X, \theta(Y))$. Then $(\cdot \mid \cdot)_{\theta}$ is an inner product on $\mathfrak{g}$. 
Lemma 1.3. $\mathfrak{g}\left(1, \operatorname{ad} Y^{0}\right) \oplus \mathfrak{g}\left(-1, \operatorname{ad} Y^{0}\right)=\mathfrak{g}(-1, \theta \tau)$.

Proof. ad $Y^{0}$ is semisimple, symmetric and with real spectrum as $Y^{0} \in \mathfrak{p}$. Hence $\mathfrak{g}=\bigoplus_{j=-1}^{1} \mathfrak{g}\left(j, \operatorname{ad} Y^{0}\right)$ is an orthogonal direct sum. But the same holds for the decomposition $\mathfrak{g}=\mathfrak{h}^{a} \oplus \mathfrak{g}(-1, \theta \tau)$. As $\mathfrak{h}^{a}=\mathfrak{g}\left(0, \operatorname{ad} Y^{0}\right)$ the lemma follows.

Let $\mathfrak{a}_{q}$ be a maximal abelian subalgebra of $\mathfrak{p}$ containing $Y^{0}$. Because of (1.3) $\mathfrak{a}_{q}$ is contained in $\mathfrak{q} \cap \mathfrak{p}$ and $\mathfrak{a}_{q}$ is abelian in $\mathfrak{q}$. We have $H=Z_{K \cap H}\left(\mathfrak{a}_{q}\right) H_{o}$, cf. $[5,16]$.

From now on we will - if not otherwise stated - assume that $\mathcal{M}$ is an irreducible semisimple symmetric space of Cayley type. Let $Y^{0}$ and $Z^{0}$ be as above. Define

$$
\mathbf{c}=\exp \frac{\pi i}{2} Y^{0} \in G_{\mathbb{C}} .
$$

Lemma 1.4. $\operatorname{Ad}(\mathbf{c}) \mid \mathfrak{g}\left(0, \operatorname{ad} Y^{0}\right)=\mathrm{id}$, and $\operatorname{Ad}(\mathbf{c}) \mid \mathfrak{g}\left( \pm 1\right.$, ad $\left.Y^{0}\right)= \pm i$ id. Furthermore $\tau \theta=\operatorname{Ad}\left(\mathbf{c}^{2}\right)=e^{\pi i \text { ad } Y^{0}}$.

Proof. The first part is obvious from the definition of $\mathbf{c}$. By Lemma 1.3 we have $\operatorname{Ad}\left(\mathbf{c}^{2}\right) \mid \mathfrak{g}(-1, \theta \tau)=-\mathrm{id}$. The claim follows now as $\mathfrak{g}\left(0, \operatorname{ad} Y^{0}\right)=\mathfrak{g}(1, \theta \tau)$.

Lemma 1.5. 1) $\operatorname{Ad}\left(\mathbf{c}^{-1}\right)=\operatorname{Ad}\left(\mathbf{c}^{3}\right)=\tau \theta \circ \operatorname{Ad}(\mathbf{c})=\operatorname{Ad}(\mathbf{c}) \circ \tau \theta$.

2) $\tau \circ \operatorname{Ad}(\mathbf{c})=\operatorname{Ad}(\mathbf{c}) \circ \theta$.

3) $\mathbf{c}^{-1} H_{\mathbb{C}} \mathbf{c}=K_{\mathbb{C}}$.

Proof. (1) This follows immediately from Lemma 1.4. For (2) we notice first that $\tau\left(Y^{0}\right)=\theta\left(Y^{0}\right)=-Y^{0}$ as $Y^{0} \in \mathfrak{q} \cap \mathfrak{p}$. Thus $\tau(\mathbf{c})=\theta(\mathbf{c})=\mathbf{c}^{-1}=\operatorname{Ad}\left(\mathbf{c}^{3}\right)$. From this we get $\tau \circ \operatorname{Ad}(\mathbf{c})=\operatorname{Ad}(\tau(\mathbf{c})) \tau=\operatorname{Ad}\left(\mathbf{c}^{-1}\right) \theta=\operatorname{Ad}(\mathbf{c}) \operatorname{Ad}(\mathbf{c})^{2} \tau=\operatorname{Ad}(\mathbf{c}) \theta$ as $\operatorname{Ad}(\mathbf{c})^{2}=\theta \tau$. (3) follows from $(2)$ as $H_{\mathbb{C}}=G_{\mathbb{C}}^{\tau}$ and $K_{\mathbb{C}}=G_{\mathbb{C}}^{\theta}$.

Lemma 1.6. Let $X^{0}=-i \operatorname{Ad}(\mathbf{c}) Z^{0}$. Then the following hold:

1) $X^{0}=\left[Y^{0}, Z^{0}\right] \in \mathfrak{h} \cap \mathfrak{p}$.

2) $\operatorname{ad}\left(X^{0}\right)$ is semisimple with spectrum $\{-1,0,1\}$.

3) $\mathfrak{h}=\mathfrak{g}\left(0, \operatorname{ad} X^{0}\right)$.

4) Let $\mathfrak{q}^{+}:=\mathfrak{g}\left(1\right.$, ad $\left.X^{0}\right)$. Then $\mathfrak{q}^{+}$is contained in $\mathfrak{q}$ and $\mathfrak{q}^{+}=\mathfrak{g} \cap \operatorname{Ad}(\mathbf{c}) \mathfrak{p}^{+}$.

5) Let $\mathfrak{q}^{-}:=\mathfrak{g}\left(-1, \operatorname{ad} X^{0}\right)$. Then $\mathfrak{q}^{-}$is contained in $\mathfrak{q}$ and $\mathfrak{q}^{-}=\mathfrak{g} \cap \operatorname{Ad}(\mathbf{c}) \mathfrak{p}^{-}$.

6) $\mathfrak{q}^{+}$and $\mathfrak{q}^{-}$are abelian Lie algebras, $\theta\left(\mathfrak{q}^{+}\right)=\mathfrak{q}^{-}$, and $\mathfrak{q}=\mathfrak{q}^{+} \oplus \mathfrak{q}^{-}$is a decomposition of $\mathfrak{q}$ into irreducible nonequivalent $H$-modules.

7) The involution $\tau$ is given by $\tau=e^{\pi i \operatorname{ad}\left(X^{0}\right)}$.

8) Let $Q^{ \pm}=\exp \mathfrak{q}^{ \pm}$. Then $P_{\max }:=H Q^{-}$is a maximal parabolic subgroup of $G$ with Levi part $H$.

Proof. (1) Let $Z^{0}=Z_{+}+Z_{-}$with $Z_{+} \in \mathfrak{g}\left(+1, \operatorname{ad} Y^{0}\right)$ and $Z_{-} \in \mathfrak{g}\left(-1, \operatorname{ad} Y^{0}\right)$. Then

$$
X^{0}=-i \operatorname{Ad}(\mathbf{c}) Z^{0}=-i\left(e^{\frac{\pi i}{2}} Z_{+}+e^{-\frac{\pi i}{2}} Z_{-}\right)=Z_{+}-Z_{-}=\left[Y^{0}, Z^{0}\right] .
$$

$\operatorname{ad}\left(X^{0}\right)$ is semisimple with real spectrum because of (1). (2) follows from the fact that ad $Z^{0}$ has spectrum $\{-i, 0, i\}$. (3)-(5) follow from Lemma 1.5 and (7) is obvious.

(6) That $\mathfrak{q}^{+}$and $\mathfrak{q}^{-}$are abelian follows from (5). That $\theta\left(\mathfrak{q}^{+}\right)=\mathfrak{q}^{-}$follows by $\theta\left(X^{0}\right)=-X^{0}$. Let $\{0\} \neq \mathfrak{s}$ be an $H$-invariant subspace in $\mathfrak{q}^{+}$. Let $\mathfrak{u}:=\left\{X \in \mathfrak{q}^{+} \mid\right.$ $\left.\forall Y \in \mathfrak{q}^{+}:(X \mid Y)_{\theta}=0\right\}, \mathfrak{q}_{1}:=\mathfrak{s} \oplus \theta(\mathfrak{s})$, and $\mathfrak{g}_{1}:=\left[\mathfrak{q}_{1}, \mathfrak{q}_{1}\right] \oplus \mathfrak{q}_{1}$. $\mathfrak{g}_{1}$ is a $\tau$ - and 
$\mathfrak{h}$-stable subalgebra of $\mathfrak{g}$ by the Jacobi identity. Furthermore $\mathfrak{u} \oplus \theta(\mathfrak{u})$ is $\mathfrak{h}$-stable. Let $X \in \mathfrak{u}$ and $Y \in \theta(s)$. Then

$$
([Y, X] \mid[Y, X])_{\theta}=-([\theta(Y),[Y, X]] \mid X)=0 .
$$

Hence $[\mathfrak{u}, \theta(s)]=\{0\}$. Applying $\theta$ to this it also follows that $[\theta(\mathfrak{u}), s]=\{0\}$. As $\left[\mathfrak{q}^{+}, \mathfrak{u}\right]=\left[\mathfrak{q}^{-}, \theta(\mathfrak{u})\right]=\{0\}$ it now follows that $\left[\mathfrak{g}_{1}, \mathfrak{u} \oplus \theta(\mathfrak{u})\right] \subset \mathfrak{g}_{1}$. Hence $\mathfrak{g}_{1}$ is a $\tau$-stable ideal in $\mathfrak{g}$. Thus $\mathbf{V}=\mathfrak{q}^{+}$.

(8) We have that $Z_{G_{\mathbb{C}}}\left(X^{0}\right)$ is connected and contained in $H_{\mathbb{C}}$ by (7). By (3) both groups have the same Lie algebra. As $H_{\mathbb{C}}$ is connected $H_{\mathbb{C}}=Z_{G_{\mathbb{C}}}\left(X^{0}\right)$. Hence $H=Z_{G}\left(X^{0}\right)$. From this the claim follows easily.

\section{The FunCtion $\Psi_{m}$}

Lemma 2.1. Let $g \in G_{\mathbb{C}}$ and $Z \in \mathfrak{p}^{+}$be such that $g \exp (-Z) \in P^{+} K_{\mathbb{C}} P^{-}$. Then $\theta(g) \exp (Z) \in P^{+} K_{\mathbb{C}} P^{-}$and $\zeta(\theta(g) \cdot Z)=-\zeta(g \cdot(-Z))$. In particular $\zeta(\theta(g))=$ $-\zeta(g)$ for $g \in P^{+} K_{\mathbb{C}} P^{-}$.

Proof. Write $g \exp -Z=\exp [g \cdot(-Z)] k p$, with $k \in K_{\mathbb{C}}$ and $p \in P^{-}$. Then

$$
\theta(g \exp -Z)=\theta(g) \exp Z=\exp (\theta(g \cdot(-Z))) k p^{-1}=\exp (-g \cdot(-Z)) k p^{-1}
$$

as $g \cdot(-Z) \in \mathfrak{p}^{+}$. Hence the lemma.

The Shilov boundary of $\mathcal{D}_{b}$ is $\mathcal{S}=G \cdot E, E:=\zeta(\mathbf{c}) \in \mathfrak{p}^{+}$. We view $G$ a subgroup of $G_{1}=G \times G$ by the diagonal embedding $g \mapsto(g, g)$. By restriction a $G_{1}$-space is therefore also a $G$-space. Similarly we view $G_{\mathbb{C}} \subset G_{1 \mathbb{C}}:=G_{\mathbb{C}} \times G_{\mathbb{C}}$.

In [7] it was proved that $\mathcal{M} \simeq G / H Q^{-} \times G / H Q^{+}$. The following lemma gives a geometric interpretation of this fact.

Lemma 2.2. 1) The stabilizer of $E$ in $G$ is $P_{\max }$.

2) We have $-E=\zeta\left(\mathbf{c}^{-1}\right)=\zeta(\theta(\mathbf{c}))$ and the stabilizer of $-E$ is $H Q^{+}$.

3) $\mathcal{S}_{1}:=\mathcal{S} \times \mathcal{S} \simeq G / H Q^{-} \times G / H Q^{+}$.

4) Let $\xi_{0}=(E,-E) \in \mathcal{S}_{1}$. Then $\mathcal{M} \simeq G \cdot \xi_{0}$ as a $G$-space.

Proof. (1) We have $H_{\mathbb{C}} Q_{\mathbb{C}}^{-} \cap G=H Q^{-}$and $g \mathbf{c} \in \mathbf{c} K_{\mathbb{C}} P^{-}$if and only if $g \in$ $\mathbf{c} K_{\mathbb{C}} P^{-} \mathbf{c}^{-1}=H_{\mathbb{C}} Q_{\mathbb{C}}^{-}$. This implies (1). (2) follows from (1) and Lemma 2.1 as $\mathbf{c}^{-1}=\theta(\mathbf{c})$ and $\theta\left(H Q^{-}\right)=H Q^{+}$. (3) and (4) are trivial in view of (1) and (2) and Lemma 2.1 .

Let $\mathfrak{t}$ be a Cartan subalgebra of $\mathfrak{k}$ (and $\mathfrak{g}$ ) such that $\mathfrak{a}:=\mathfrak{t} \cap \mathfrak{q}$ is maximal abelian in $\mathfrak{q}$, cf. [17]. Then $Z^{0} \in \mathfrak{a}$. Let $\Delta=\Delta\left(\mathfrak{g}_{\mathbb{C}}, \mathfrak{t}_{\mathbb{C}}\right)$ be the set of roots of $\mathfrak{t}_{\mathbb{C}}$ in $\mathfrak{g}$. Then

$$
\begin{aligned}
\Delta_{k} & :=\left\{\alpha \in \Delta \mid \alpha\left(Z^{0}\right)=0\right\}=\Delta\left(\mathfrak{k}_{\mathbb{C}}, \mathfrak{t}_{\mathbb{C}}\right), \\
\Delta_{n} & :=\left\{\alpha \in \Delta \mid \alpha\left(Z^{0}\right)= \pm i\right\}=\Delta\left(\mathfrak{p}_{\mathbb{C}}, \mathfrak{t}_{\mathbb{C}}\right) .
\end{aligned}
$$

Choose a positive system $\Delta_{k}^{+}$and let $\Delta^{+}:=\Delta_{n}^{+} \cup \Delta_{k}^{+}$, where $\Delta_{n}^{+}=\left\{\alpha \mid \mathfrak{g}_{\mathbb{C} \alpha} \subset \mathfrak{p}^{+}\right\}$. Let $\Gamma=\left\{\gamma_{1}, \ldots, \gamma_{r}\right\}$ be a maximal set of strongly orthogonal roots in $\Delta_{n}^{+}$such that $\gamma_{1}$ is the maximal root in $\Delta_{n}^{+}$and $\gamma_{j+1}$ is the maximal root strongly orthogonal to $\gamma_{1}, \ldots, \gamma_{j}$. By the Theorem of Moore [8] and [17] we have the following facts:

Lemma 2.3. 1) Let $H_{1}, \ldots, H_{r} \in i \mathfrak{a}$ be given by $H_{j} \in\left[\mathfrak{g}_{\mathbb{C} \gamma_{j}}, \mathfrak{g}_{\mathbb{C}\left(-\gamma_{j}\right)}\right]$ and $\gamma_{j}\left(H_{j}\right)=2$. Then $\mathfrak{a}=i \bigoplus_{j=1}^{r} \mathbb{R} H_{j}$.

2) $Z^{0}=\frac{i}{2} \sum_{j=1}^{r} H_{j}$. 
3) We can choose $\Delta_{k}^{+}$and $\Delta_{n}^{+}$such that

$\Delta_{n}^{+}=\left\{\frac{1}{2}\left(\gamma_{i}+\gamma_{j}\right) \mid 1 \leq i \leq j \leq r\right\} \quad$ and $\quad \Delta_{k}^{+}=\left\{\frac{1}{2}\left(\gamma_{i}-\gamma_{j}\right) \mid 1 \leq i<j \leq r\right\}$.

4) The root spaces $\mathfrak{g}_{\mathbb{C} \gamma_{j}}$ are one dimensional and all the root spaces corresponding to a root of the form $\frac{1}{2}\left(\gamma_{i} \pm \gamma_{j}\right)$ have the same dimension $d$.

By Proposition 4.2.11 in [5] we have

Lemma 2.4. Suppose that $C$ is generated by $-\operatorname{Ad}(H) Z^{0}$. Then $i\left[C^{o} \cap \mathfrak{a}\right]=$ $\left\{\sum_{j=1}^{r} t_{j} H_{j} \mid t_{j}>0\right\}$.

We list here the irreducible symmetric spaces $G / H$ of Cayley type, cf. [5], the Shilov boundary of $G / K$, together with the real rank $r$ of $G / K$, and the common dimension $d$. Here $k \geq 3, \mathbf{T}$ is the one dimensional torus, $Q_{n}$ is the real quadric in the real projective space $\mathbb{R P}^{n}$ defined by the quadratic form of signature $(1, n)$ and the subscript + means positive determinant:

\section{Irreducible Spaces of Cayley Type}

\begin{tabular}{llcc}
\multicolumn{1}{c}{$\mathcal{M}=G / H$} & $\mathcal{S}=K / K \cap H$ & $r$ & $d$ \\
\hline $\mathrm{Sp}(n, \mathbb{R}) / \mathrm{GL}(n, \mathbb{R})_{+}$ & $U(n) / O(n)$ & $n$ & 1 \\
$\mathrm{SU}(n, n) / \mathrm{GL}(n, \mathbb{C})_{+}$ & $U(n)$ & $n$ & 2 \\
$\mathrm{SO}^{*}(4 n) / \mathrm{SU}^{*}(2 n) \mathbb{R}^{+}$ & $U(2 n) / \mathrm{Sp}(2 n)$ & $2 n$ & 4 \\
$\mathrm{SO}(2, k) / \mathrm{SO}(1, k-1) \mathbb{R}^{+}$ & $Q_{n}$ & 2 & $k-2$ \\
$E_{7(-25)} / E_{6(-26)} \mathbb{R}^{+}$ & $E_{6} \mathbf{T} / F_{4}$ & 3 & 8
\end{tabular}

The following lemma and theorem are well known, but we prove them here for lack of a good reference.

Lemma 2.5. Let $\rho_{n}$ be the half sum of positive noncompact roots with multiplicities.

1) $\rho_{n}=\frac{1}{2}\left(1+\frac{d(r-1)}{2}\right)\left(\gamma_{1}+\cdots+\gamma_{r}\right)$.

2) $\frac{2\left(\rho_{n} \mid \gamma_{j}\right)}{\left|\gamma_{j}\right|^{2}}=1+\frac{d(r-1)}{2}$ and $\frac{2\left(\rho_{n} \mid \frac{1}{2}\left(\gamma_{i}+\gamma_{j}\right)\right)}{\left|\frac{1}{2}\left(\gamma_{i}+\gamma_{j}\right)\right|^{2}}=2+d(r-1)$.

3) $\frac{2\left(\rho_{n} \mid \gamma_{j}\right)}{\left|\gamma_{j}\right|^{2}} \in \mathbb{Z}^{+}$if and only if $\mathfrak{g} \neq \mathfrak{s p}(2 n, \mathbb{R})$ or $\mathfrak{g} \neq \mathfrak{s o}(2,2 n+1)$.

4) $\gamma_{1}$ is a long root.

Proof. (1) follows from Lemma 2.3, part 3. (2) is a direct consequence of (1), and (3) is clear from the last column of the above list of Cayley type spaces. (4) follows by inspection of the Dynkin diagrams.

Theorem 2.6. Fix the positive system $\Delta^{+}\left(\mathfrak{g}_{\mathbb{C}}, \mathfrak{t}_{\mathbb{C}}\right)=\Delta_{n}^{+} \cup-\Delta_{k}^{+}$. Then the following hold:

1) There exists an irreducible finite dimensional representation of $G_{\mathbb{C}}$ with lowest weight $-2 \rho_{n}$.

2) Assume that $\mathfrak{g} \neq \mathfrak{s p}(2 n, \mathbb{R}), \mathfrak{s o}(2,2 k+1), n, k \geq 1$. Then there exists a finite dimensional irreducible representation of $G_{\mathbb{C}}$ with lowest weight $-\rho_{n}$.

Proof. Let $\left\{\alpha_{1}, \ldots, \alpha_{k}\right\}$ be the simple roots for $\Delta^{+}\left(\mathfrak{g}_{\mathbb{C}}, \mathfrak{t}_{\mathbb{C}}\right)$. Then $\alpha_{1}=\gamma_{1}$ is the unique simple noncompact root. Furthermore $\left(\rho_{n} \mid \alpha\right)=0$ for $\alpha \in \Delta_{k}$. If $\gamma$ is an 
arbitrary noncompact positive root, then $\gamma=\gamma_{1}+\sum_{j>1} n_{\alpha_{j}} \alpha_{j}$. By Lemma 2.5:

$$
\frac{2\left(k \rho_{n} \mid \gamma\right)}{|\gamma|^{2}}=\frac{2\left(k \rho_{n} \mid \gamma_{1}\right)}{\left|\gamma_{1}\right|^{2}} \frac{\left|\gamma_{1}\right|^{2}}{|\gamma|^{2}}=k\left(1+\frac{d(r-1)}{2}\right) \frac{\left|\gamma_{1}\right|^{2}}{|\gamma|^{2}} \text {. }
$$

As $\gamma_{1}$ is a long root $\left|\gamma_{1}\right|^{2} /|\gamma|^{2} \in \mathbb{Z}^{+}$. But $\left(1+\frac{d(r-1)}{2}\right) \in \mathbb{Z}$ if and only if $d$ is even or $r$ is odd, and in all cases $2\left(1+\frac{d(r-1)}{2}\right) \in \mathbb{Z}$. The claim now follows from the list of Cayley type space above.

Let $m \in \mathbb{Z}^{+}$be such that there exists a finite dimensional irreducible representation $\left(\pi_{m}, \mathbf{V}_{m}\right)$ of $G_{\mathbb{C}}$ with lowest weight $-m \rho_{n}$. Choose an inner product on $\mathbf{V}_{m}$ such that $\pi_{m}$ is unitary on the maximal compact subgroup of $G_{\mathbb{C}}$ corresponding to the Lie algebra $\mathfrak{k} \oplus i \mathfrak{p}$. Let $\sigma: G_{\mathbb{C}} \rightarrow G_{\mathbb{C}}$ be the involutive conjugation with differential $X+i Y \mapsto X-i Y, X, Y \in \mathfrak{g}$. Then

$$
\pi_{m}(g)^{*}=\pi_{m}\left(\sigma \theta(g)^{-1}\right) .
$$

Let $u_{0}$ be a lowest weight vector of norm 1 , and let $\mathbf{c}$ be as before. Define $\Phi_{m}$ : $\mathfrak{p}^{+} \rightarrow \mathbb{C}$ and $\Psi_{m}: \mathfrak{p}^{+} \times \mathfrak{p}^{+} \rightarrow \mathbb{C}$ by

$$
\Phi_{m}(Z):=\left(\pi_{m}\left(\mathbf{c}^{-2} \exp Z\right) u_{0} \mid u_{0}\right) \quad \text { and } \quad \Psi_{m}(Z, W):=\Phi_{m}(Z-W) .
$$

Let $G=\mathrm{SU}(1,1) \subset G_{\mathbb{C}}=\mathrm{SL}(2, \mathbb{C})$. Let $\tau(X)=\bar{X}$. Then $\tau=\operatorname{Ad}\left(\begin{array}{ll}0 & 1 \\ 1 & 0\end{array}\right)$ and the holomorphic extension of $\tau$ is given by

$$
\tau\left(\left(\begin{array}{cc}
a & b \\
c & -a
\end{array}\right)\right)=\left(\begin{array}{cc}
-a & c \\
b & a
\end{array}\right)
$$

With $Z^{0}=\frac{i}{2}\left(\begin{array}{cc}1 & 0 \\ 0 & -1\end{array}\right)$ we have

$$
\mathfrak{p}^{+}=\left\{\left(\begin{array}{ll}
0 & z \\
0 & 0
\end{array}\right) \mid z \in \mathbb{C}\right\} \quad \text { and } \quad \mathfrak{p}^{-}=\left\{\left(\begin{array}{cc}
0 & 0 \\
w & 0
\end{array}\right) \mid w \in \mathbb{C}\right\} .
$$

The Cartan involution $\theta$ is given by conjugating by $Z^{0}$ and the holomorphic extension to $\mathrm{SL}(2, \mathbb{C})$ is

$$
\left(\begin{array}{ll}
a & b \\
c & d
\end{array}\right) \mapsto\left(\begin{array}{cc}
a & -b \\
-c & d
\end{array}\right)
$$

Thus $\tau \theta(X)=-X^{T}$. Identify $\mathfrak{p}^{+}$and $\mathfrak{k}_{\mathbb{C}}$ with $\mathbb{C}$ by $z \mapsto\left(\begin{array}{ll}0 & z \\ 0 & 0\end{array}\right)$ respectively $\left(\begin{array}{cc}w & 0 \\ 0 & -w\end{array}\right) \mapsto w$ and $K_{\mathbb{C}} \simeq \mathbb{C}^{*}$. A simple calculation now shows that

$$
\left(\begin{array}{ll}
a & b \\
c & d
\end{array}\right)=\left(\begin{array}{cc}
1 & b / d \\
0 & 1
\end{array}\right)\left(\begin{array}{cc}
1 / d & 0 \\
0 & d
\end{array}\right)\left(\begin{array}{cc}
1 & 0 \\
c / d & 1
\end{array}\right)
$$

if $d \neq 0$. Thus $P^{+} K_{\mathbb{C}} P^{-}=\left\{\left(\begin{array}{ll}a & b \\ c & d\end{array}\right) \mid d \neq 0\right\}$, and $\zeta\left(\left(\begin{array}{ll}a & b \\ c & d\end{array}\right)\right)=\frac{b}{d}$. We have $\mathcal{D}_{b}=\{z \in \mathbb{C}|| z \mid<1\}$ and $\mathcal{S}=\{z \in \mathbb{C}|| z \mid=1\}$. Furthermore $k\left(\left(\begin{array}{ll}a & b \\ c & d\end{array}\right)\right)=$ $1 / d$. Thus we recover the well known facts:

$$
\left(\begin{array}{ll}
a & b \\
c & d
\end{array}\right) \cdot z=\frac{a z+b}{c z+d} \text { and } k\left(\left(\begin{array}{ll}
a & b \\
c & d
\end{array}\right), z\right)=(c z+d)^{-1}
$$


Let $Y^{0}=\frac{i}{2}\left(\begin{array}{cc}0 & -1 \\ 1 & 0\end{array}\right)$. Then $X^{0}=-\frac{1}{2}\left(\begin{array}{ll}0 & 1 \\ 1 & 0\end{array}\right)$. Thus

$$
\mathfrak{q}^{+}=\left\{i\left(\begin{array}{cc}
r & r \\
-r & -r
\end{array}\right) \mid r \in \mathbb{R}\right\} \quad \text { and } \quad \mathfrak{q}^{-}=\left\{i\left(\begin{array}{cc}
r & -r \\
r & -r
\end{array}\right) \mid r \in \mathbb{R}\right\} \text {. }
$$

Furthermore $\exp t i Y^{0}=\left(\begin{array}{cc}\cos \frac{t}{2} & \sin \frac{t}{2} \\ -\sin \frac{t}{2} & \cos \frac{t}{2}\end{array}\right)$ which implies that $\mathbf{c}=\frac{1}{\sqrt{2}}\left(\begin{array}{cc}1 & 1 \\ -1 & 1\end{array}\right)$ and $\mathbf{c}^{2}=\left(\begin{array}{cc}0 & 1 \\ -1 & 0\end{array}\right)$. Hence

Lemma 2.7. Let $G=\mathrm{SU}(1,1)$. Let $Z=\left(\begin{array}{ll}1 & z \\ 0 & 1\end{array}\right) \in \mathfrak{p}^{+}$. Then $\mathbf{c}^{-2} \exp Z=$ $\left(\begin{array}{cc}0 & -1 \\ 1 & z\end{array}\right)$ and $\mathbf{c}^{-2} \exp Z \in P^{+} K_{\mathbb{C}} P^{-}$if and only if $z \neq 0$. Furthermore $\zeta(\mathbf{c})=1$, $\zeta\left(\mathbf{c}^{-1}\right)=-1$, and $k\left(\mathbf{c}^{-2}, z\right)=1 / z$.

Lemma 2.8. Let $G=\mathrm{SU}(1,1)$. Then $\Phi_{m}(z)=z^{m}$ and $\Psi_{m}(z, w)=(z-w)^{m}$.

In general we choose $E_{j} \in \mathfrak{g}_{\mathbb{C} \gamma_{j}}, j=1, \ldots, r$, such that $H_{j}=\left[E_{j}, E_{-j}\right]$, $E_{-j}=\tau\left(E_{j}\right)=\sigma\left(E_{j}\right)$, and $Y^{0}=-\frac{i}{2} \sum_{j=1}^{r}\left(E_{j}-E_{-j}\right)$. For $j=1, \ldots, r$, define a homomorphism $\varphi_{j}: \mathfrak{s l}(2, \mathbb{C}) \rightarrow \mathfrak{g}_{\mathbb{C}}$ by

$$
\left(\begin{array}{cc}
1 & 0 \\
0 & -1
\end{array}\right) \mapsto H_{j}, \quad\left(\begin{array}{cc}
0 & 1 \\
0 & 0
\end{array}\right) \mapsto E_{j} \quad \text { and } \quad\left(\begin{array}{cc}
0 & 0 \\
1 & 0
\end{array}\right) \mapsto E_{-j} .
$$

The conjugation of $\mathfrak{s l}(2, \mathbb{C})$ with respect to $\mathfrak{s u}(1,1)$ is given by

$$
\sigma_{s u(1,1)}: X \mapsto-\left(\begin{array}{cc}
1 & 0 \\
0 & -1
\end{array}\right) X^{*}\left(\begin{array}{cc}
1 & 0 \\
0 & -1
\end{array}\right)
$$

Thus $\varphi_{j} \circ \sigma_{s u(1,1)}=\sigma \circ \varphi_{j}$ from which it follows that $\varphi_{j}(\mathfrak{s u}(1,1)) \subset \mathfrak{g}$. Furthermore $\varphi_{j}$ intertwines the involutions $\theta, \tau$ and $\tau \theta$ on $\mathfrak{s u}(1,1)$ and on $\mathfrak{g}$. We denote the corresponding homomorphism $\mathrm{SL}(2, \mathbb{C}) \rightarrow G_{\mathbb{C}}$ also by $\varphi_{j}$. As the roots $\gamma_{j}$ are strongly orthogonal $\mathfrak{g}_{j}:=\varphi_{j}(\mathfrak{s u}(1,1))$ commutes with $\mathfrak{g}_{k}$ if $j \neq k$. Using $\varphi_{j}$ calculations involving the strongly orthogonal roots and the algebras $\mathfrak{g}_{j}$ are reduced to calculations in $\mathfrak{s l}(2, \mathbb{C})$ and $\mathrm{SL}(2, \mathbb{C})$. In particular we get $E=\sum E_{j}$.

Let $\xi$ be a character of $K_{\mathbb{C}}$ and assume it is unitary on $K$. Then $d \xi \in i_{\mathfrak{z}}(\mathfrak{k})$. We write $k^{d \xi}:=\xi(k), k \in K_{\mathbb{C}}$. The following is well known:

Lemma 2.9. Let $(\pi, \mathbf{V})$ be a finite dimensional and irreducible representation of $G_{\mathbb{C}}$ with lowest weight $\mu$. Let $u_{o}$ be a non-zero vector of weight $\mu$. Then the $K_{\mathbb{C} \text { - }}$ module generated by $u_{o}$ is irreducible. If $\mu \in i_{\mathfrak{z}}(\mathfrak{k})^{*}$, then $K_{\mathbb{C}}$ acts on $\mathbb{C} u_{o}$ by the character $k \mapsto k^{\mu}$.

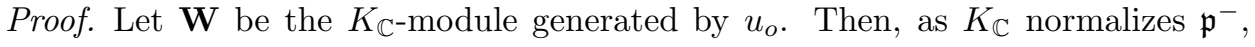
$\mathbf{W} \subset \mathbf{V}^{\mathfrak{p}^{-}}$. Here $\mathbf{V}$ is the representation space of $\pi$. Let $\mathfrak{n}_{\mathbb{C}}^{-}=\bigoplus_{\alpha \in \Delta_{k}^{+}} \mathfrak{k}_{\mathbb{C} \alpha}$. Then $\mathbf{W}^{\mathfrak{n}_{\mathbb{C}}^{-}} \subset \mathbf{V}^{\mathfrak{n}_{\mathbb{C}}^{-} \oplus \mathfrak{p}^{-}}$. But $\mathbf{V}^{\mathfrak{n}_{\mathbb{C}}^{-} \oplus \mathfrak{p}^{-}}=\mathbb{C} u_{o}$. Thus $\mathbf{W}^{\mathfrak{n}_{\mathbb{C}}^{-}}$is one-dimensional and the claim follows.

Lemma 2.10. Let $\sigma: G_{\mathbb{C}} \rightarrow G_{\mathbb{C}}$ be the conjugation with respect to $G$. Let $\chi$ : $K_{\mathbb{C}} \rightarrow \mathbb{C}^{*}$ be a character. If $\chi \mid K$ is unitary, then $\overline{\chi(\sigma \tau(k))}=\chi(k)$.

Proof. Both sides are holomorphic in $k$ and agree on $K$ as $\tau \mid \mathfrak{z}(\mathfrak{k})=-1$. The lemma now follows as $K$ is a real form of $K_{\mathbb{C}}$. 
Lemma 2.11. Let $Z \in \mathfrak{p}^{+}$. Then the following holds:

1) If $\exp Z \in \mathbf{c}^{2} P^{+} K_{\mathbb{C}} P^{-}$, then

$$
\Phi_{m}(Z)=\left(\pi_{m}\left(k\left(\mathbf{c}^{-2}, Z\right)\right) u_{0} \mid u_{0}\right)=k\left(\mathbf{c}^{-2}, Z\right)^{-m \rho_{n}} .
$$

2) $\Phi_{m}(k \cdot Z)=k^{2 m \rho_{n}} \Phi_{m}(Z)$ for every $k \in K_{\mathbb{C}}$.

3) Let $z_{1}, \ldots, z_{r} \in \mathbb{C}$. Then

$$
\Phi_{m}\left(\operatorname{Ad}(k) \sum_{j=1}^{r} z_{j} E_{j}\right)=k^{2 m \rho_{n}} \prod_{j=1}^{n} z_{j}^{m(1+d(r-1) / 2)} .
$$

Proof. (1) Write $\mathbf{c}^{-2} \exp Z=p k q$ with $p \in P^{+}, k=k\left(\mathbf{c}^{-2}, Z\right)$, and $q \in P^{-}$. As $\sigma\left(\mathfrak{p}^{+}\right)=\mathfrak{p}^{-}$we get $\pi_{m}(p)^{*} u_{0}=\pi_{m}\left(\sigma \theta(p)^{-1}\right) u_{0}=u_{0}$. Thus

$$
\begin{aligned}
\Phi_{m}(Z) & =\left(\pi_{m}\left(\mathbf{c}^{-2} \exp Z\right) u_{0} \mid u_{0}\right) \\
& =\left(\pi_{m}(k) \pi_{m}(q) u_{0} \mid \pi_{m}(p)^{*} u_{0}\right) \\
& =\left(\pi_{m}(k) u_{0} \mid u_{0}\right) \\
& =k^{-m \rho_{n}}
\end{aligned}
$$

where the last equality follows from Lemma 2.9

(2) We have $k \cdot Z=\operatorname{Ad}(k) Z$. By Lemma 2.9:

$$
\begin{aligned}
& \Phi_{m}(k \cdot Z)=\left(\pi_{m}\left(\mathbf{c}^{-2} k \exp Z k^{-1}\right) u_{0} \mid u_{0}\right) \\
& =\left(\pi_{m}(\tau(k)) \pi_{m}\left(\mathbf{c}^{-2} \exp Z\right) \pi_{m}\left(k^{-1}\right) u_{0} \mid u_{0}\right) \\
& =k^{m \rho_{n}}\left(\pi_{m}\left(\mathbf{c}^{-2} \exp Z\right) u_{0} \mid \pi_{m}\left(\sigma \tau(k)^{-1}\right) u_{0}\right) \\
& =k^{m \rho_{n}} \overline{(\sigma \tau(k))^{m \rho_{n}}} \Phi_{m}(Z) \\
& =k^{2 m \rho_{n}} \Phi_{m}(Z) \text {. }
\end{aligned}
$$

(3) This follows from (2) and $\mathfrak{s u}(1,1)$-reduction.

Theorem 2.12. 1) Let $g \in G_{\mathbb{C}}$ and $Z, W \in \mathfrak{p}^{+}$be such that $g \cdot Z$ and $g \cdot W$ are defined. Then $\Psi_{m}(g \cdot Z, g \cdot W)=k(g, Z)^{m \rho_{n}} k(g, W)^{m \rho_{n}} \Psi_{m}(Z, W)$.

2) Let $z_{j}, w_{j} \in \mathbb{C}, j=1, \ldots, r$, and let $k \in K_{\mathbb{C}}$. Then

$$
\Psi_{m}\left(k \cdot \sum_{j=1}^{r} z_{j} E_{j}, k \cdot \sum_{j=1}^{r} w_{j} E_{j}\right)=k^{2 m \rho_{n}} \prod_{j=1}^{r}\left(z_{j}-w_{j}\right)^{m(1+d(r-1) / 2)} .
$$

Proof. (1) By definition $\exp g \cdot Z=g \exp Z k(g, Z)^{-1} q, q \in P^{-}$, and similarly for $W$. Thus

$$
\begin{aligned}
\exp (g \cdot Z-g \cdot W) & =\exp (-g \cdot W) \exp (g \cdot Z) \\
& =\left(g \exp (W) k(g, W)^{-1} p\right)^{-1} g \exp (Z) k(g, Z)^{-1} q \\
& =p^{-1} k(g, W) \exp (-W) \exp (Z) k(g, Z)^{-1} q
\end{aligned}
$$

for some $p \in P^{-}$. Now $\operatorname{Ad}\left(\mathbf{c}^{-2}\right)=\theta \tau$. Thus $\mathbf{c}^{-2} P^{-} \mathbf{c}^{2}=P^{+}$. As above we get

$$
\begin{aligned}
\Psi_{m}(g \cdot Z, g \cdot W) & =\left(\pi_{m}\left(\mathbf{c}^{-2} p^{-1} k(g, W) \exp (-W) \exp (Z) k(g, Z)^{-1}\right) u_{0} \mid u_{0}\right) \\
& =k(g, Z)^{m \rho_{n}} k(g, W)^{m \rho_{n}} \Psi_{m}(Z, W) .
\end{aligned}
$$

(2) We have $k \cdot \sum_{j} z_{j} E_{j}-k \cdot \sum_{j} w_{j} E_{j}=k \sum_{j}\left(z_{j}-w_{j}\right) E_{j}$ as $K_{\mathbb{C}}$ acts by linear transformations. The claim follows now from Lemma 2.11. 


\section{The Causal Compactification of $\mathcal{M}$}

In this section we show that the map

$$
\mathcal{M} \ni g H \mapsto g \cdot(E,-E) \in\left\{\xi \in \mathcal{S}_{1} \mid \Psi_{m}(\xi) \neq 0\right\} \subset \mathcal{S}_{1}
$$

is a causal compactification of $\mathcal{M}$. Recall that the causal structure on $\mathcal{M}$ is determined by the cone $C$ in $\mathfrak{q}$ generated by $-\operatorname{Ad}(H) Z^{0}$, cf. $[5,14,15]$. The cone $C$ can also be described in the following way, cf. $[5,14]$ :

Lemma 3.1. Write $Y^{0}=Y^{+}-Y^{-}$with $Y^{ \pm} \in \mathfrak{q}^{ \pm H \cap K}$. Let $C_{+}$be the $H$-invariant cone in $\mathfrak{q}^{+}$given by $C_{+}=\operatorname{Ad}(H) Y^{+}$. Then the following hold:

1) $-Z^{0}=Y^{+}+Y^{-}$.

2) $C_{+}$is regular and self-dual.

3) Let $C^{-}=\theta\left(C^{+}\right)=\operatorname{Ad}(H) Y^{-}$. Then $C=C_{+}+C_{-}$.

Proof. (1) is a standard $\mathfrak{s u}(1,1)$-reduction. (2) and (3) are from [5], Theorem 2.6.8.

Identify the tangent space of $\mathcal{S}_{1}$ at $\xi_{0}$ with $(\mathfrak{g} \times \mathfrak{g}) /\left(\left(\mathfrak{h}+\mathfrak{q}^{-}\right) \times\left(\mathfrak{h}+\mathfrak{q}^{+}\right)\right) \simeq \mathfrak{q}^{+} \times \mathfrak{q}^{-}$. Let $D$ be the image of $C_{+} \times C_{-}$in $T_{\xi_{0}} \mathcal{S}_{1}$ under this identification. Then $D$ is an $H \times H$-invariant regular cone in $T_{\xi_{0}}\left(\mathcal{S}_{1}\right)$.

Lemma 3.2. $D$ is an $\left(H Q^{-}\right) \times\left(H Q^{+}\right)$-invariant cone in $T_{\xi_{0}}\left(\mathcal{S}_{1}\right)$.

Proof. Let $q=\exp X \in Q^{+}$and $p=\exp Y \in Q^{-}$. Let $(R, T) \in \mathfrak{q}^{+} \times \mathfrak{q}^{-}$. Then

$$
\begin{aligned}
\operatorname{Ad}(p, q)(R, T) & =\left(e^{\operatorname{ad} Y} R, e^{\operatorname{ad} X} T\right) \\
& =(R+[Y, R]+[Y,[Y, R]], T+[X, T]+[X,[X, T]]) .
\end{aligned}
$$

But $[Y, R] \in \mathfrak{h}$ and $[Y,[Y, R]] \in \mathfrak{q}^{-}$. Thus $R+[Y, R]+[Y,[Y, R]]=R \bmod (\mathfrak{h}+$ $\left.\mathfrak{q}^{-}\right)$. Similarly $T+[X, T]+[X,[X, T]]=T \bmod \left(\mathfrak{h}+\mathfrak{q}^{+}\right)$. It follows that $\operatorname{Ad}(p, q)$ restricted to $T_{\xi_{0}}\left(\mathcal{S}_{1}\right)$ is the identity map. By this the claim follows.

As explained in Section 1 it now follows that $D$ defines a $G \times G$-invariant causal structure on $\mathcal{S}_{1}$ by translating the cone $D$ by $G \times G$.

Let $Y_{ \pm j}=-i \operatorname{Ad}(\mathbf{c}) E_{ \pm j}, j=1, \ldots, r$. In the case of $\mathfrak{s u}(1,1)$ we have

$$
Y_{1}=-\frac{i}{2}\left(\begin{array}{cc}
1 & 1 \\
-1 & -1
\end{array}\right) \in \mathfrak{q}^{+}, \quad Y_{-1}=-\theta\left(Y_{1}\right) \in \mathfrak{q}^{+}, \quad \text { and } \quad Y^{0}=\frac{1}{2}\left(Y_{1}-Y_{-1}\right) .
$$

Lemma 3.3. Let $j=1, \ldots, r$. Then

1) $Y_{j} \in \mathfrak{q}^{+}$and $Y_{-j}=\theta\left(Y_{j}\right) \in \mathfrak{q}^{-}$.

2) $Y^{0}=\frac{1}{2} \sum_{j=1}^{r}\left(Y_{j}-Y_{-j}\right)$, i.e., $Y^{+}=2 \sum_{j=1}^{r} Y_{j}$ and $Y^{-}=2 \sum_{j=1}^{r} Y_{-j}$.

3) $Y_{ \pm k} \in C$.

Proof. (1) By Lemma $1.4 \tau \circ \operatorname{Ad}(\mathbf{c})=\operatorname{Ad}(\mathbf{c}) \circ \theta$. Thus $\tau\left(Y_{j}\right)=-i \tau\left(\operatorname{Ad}(\mathbf{c}) E_{j}\right)=$ $-i \operatorname{Ad}(\mathbf{c}) \theta\left(E_{j}\right)=-Y_{j}$, as $\theta\left(E_{j}\right)=-E_{j}$. From $\sigma \circ \operatorname{Ad}(\mathbf{c})=\operatorname{Ad}\left(\mathbf{c}^{-1}\right) \circ \sigma$ we get $\sigma\left(Y_{j}\right)=i \operatorname{Ad}(\mathbf{c}) \theta \tau\left(\sigma\left(E_{j}\right)\right)=i \operatorname{Ad}(\mathbf{c})\left(-E_{j}\right)=Y_{j}$. This shows that $Y_{j} \in \mathfrak{q}$. Furthermore $\left[X^{0}, Y_{j}\right]=-\operatorname{Ad}(\mathbf{c})\left[Z^{0}, E_{j}\right]=-i \operatorname{Ad}(\mathbf{c}) E_{j}=Y_{j}$. Hence $Y_{j} \in \mathfrak{q}^{+}$. As $\theta \operatorname{Ad}(\mathbf{c})=\operatorname{Ad}(\mathbf{c}) \tau$ we get $\theta\left(Y_{j}\right)=-i \operatorname{Ad}(\mathbf{c}) \tau\left(E_{j}\right)=-i \operatorname{Ad}(\mathbf{c})\left(E_{-j}\right)=Y_{-j}$.

(2) This follows by equation (3.1), Lemma 3.1, and $\mathfrak{s u}(1,1)$-reduction. 
(3) Let $X_{j}^{0}=\varphi_{j}\left(-\frac{1}{2}\left(\begin{array}{ll}0 & 1 \\ 1 & 0\end{array}\right)\right) \in \mathfrak{h} \cap \mathfrak{p}$. Then an $\mathfrak{s u}(1,1)$-reduction shows that

$$
\operatorname{Ad}\left(\exp t X_{j}^{0}\right) Y_{k}=\left\{\begin{array}{cc}
Y_{k}, & k \neq j, \\
e^{t} Y_{k}, & k=j
\end{array}\right.
$$

and

$$
\operatorname{Ad}\left(\exp t X_{j}^{0}\right) Y_{-k}=\left\{\begin{array}{cc}
Y_{-k}, & k \neq j \\
e^{-t} Y_{-k}, & k=j
\end{array}\right.
$$

In particular

$$
\lim _{t \rightarrow \infty} e^{-t} e^{t \text { ad } X_{j}^{0}} \sum_{k=1}^{r}\left(Y_{k}+Y_{-k}\right)=Y_{j} \quad \text { and } \quad \lim _{t \rightarrow-\infty} e^{t} e^{\operatorname{ad} t X_{j}^{0}} \sum_{k=1}^{r}\left(Y_{k}+Y_{-k}\right)=Y_{-j} .
$$

The claim follows now from (2) and Lemma 3.1.

We will also need the following lemma, cf. [3] or [5]:

Lemma 3.4. Let $\mathfrak{a}_{q}:=\sum_{j=1}^{r} \mathbb{R}\left(Y_{j}-Y_{-j}\right)$. Then $\mathfrak{a}_{q}$ is a maximal abelian subalgebra of $\mathfrak{p}$ contained in $\mathfrak{q} \cap \mathfrak{p}$.

Lemma 3.5. $\mathfrak{q}^{+}=\operatorname{Ad}(H \cap K) \sum_{j=1}^{r} \mathbb{R} Y_{j}$.

Proof. Let $X \in \mathfrak{q}^{+}$. Then $X-\theta(X) \in \mathfrak{q} \cap \mathfrak{p}$. Therefore we can find $k \in K \cap H$ and $x_{j} \in \mathbb{R}$ such that $\operatorname{Ad}(k)(X-\theta(X))=\sum_{j=1}^{r} x_{j}\left(Y_{j}-Y_{-j}\right) \in \mathfrak{a}_{q}$. As $\theta(X), Y_{-j} \in \mathfrak{q}^{-}$ it follows that $\operatorname{Ad}(k) X=\sum_{j=1}^{r} x_{j} Y_{j}$.

Lemma 3.6. $Q^{+} \cdot E=\operatorname{Ad}(K \cap H)\left\{\sum_{j=1}^{r} z_{j} E_{j} \in \mathcal{S}|| z_{j} \mid=1, z_{j} \neq-1\right\}$.

Proof. Let $X=\operatorname{Ad}(k) \sum_{j=1}^{r} r_{j} Y_{j} \in \mathfrak{q}$ with $r_{j} \in \mathbb{R}$. Then a simple $\mathfrak{s u}(1,1)$ calculation shows that $\exp X \cdot E=\operatorname{Ad}(k) \sum_{j=1}^{r} \frac{1-i r_{j}}{1+i r_{j}} E_{j}$. But $\frac{1-i r}{1+i r}=z, z \in \mathbb{C}$ has a solution if and only if $z \neq-1$. The solution $r$ is then given by $\frac{1}{i} \frac{1-z}{1+z}$.

Corollary 3.7. $\mathcal{S}=(K \cap H)\left\{\sum_{j=1}^{r} z_{j} E_{j} \in \mathcal{S}|| z_{j} \mid=1\right\}$.

Proof. By Lemma 3.6 we have $Q^{+} \cdot E \subset(K \cap H)\left\{\sum_{j=1}^{r} z_{j} E_{j} \in \mathcal{S}|| z_{j} \mid=1\right\}$ and the second set is closed in $\mathcal{S}$. On the other hand $Q^{+} \cdot E$ is dense in $\mathcal{S}$ as $Q^{+} H Q^{-}$ is dense in $G$.

Theorem 3.8. Let $m \in \mathbb{N}$ be such that $\Psi_{m}$ is defined. Define $\Phi: \mathcal{M} \rightarrow \mathcal{S}_{1}$ by $\Phi(g H):=g \cdot \xi_{0}$. Then $\left(\mathcal{S}_{1}, \Phi\right)$ is a G-equivariant, causal compactification of $\mathcal{M}$ with $\Phi(\mathcal{M})=\left\{\xi \in \mathcal{S}_{1} \mid \Psi_{m}(\xi) \neq 0\right\}$.

Proof. The function $\Phi$ is by definition $G$-equivariant. As the causal structure on $\mathcal{M}$ and $\mathcal{S}_{1}$ are $G$-invariant we only have to show that $(d \Phi)_{\mathbf{o}}(C) \subset D_{\xi_{0}}$. But this is obvious from the definition of $D$. To show that the image is dense it suffices to show that it is given as stated. It follows by Theorem 2.12 that the left-hand side is contained in the right-hand side. Assume now that $\Psi_{m}(Z, W) \neq 0,(Z, W) \in \mathcal{S}_{1}$. Let $g \in G$ be such that $g \cdot W=-E$ and then choose $k \in K \cap H$ such that $k \cdot(g \cdot Z)=\sum z_{j} E_{j}$. Then $(k g) \cdot \xi=\left(\sum_{j=1}^{r} z_{j} E_{j},-E\right)$. By Theorem 2.12 we have $\Psi_{m}((k g) \cdot \xi) \neq 0$. By part 2 of that theorem $z_{j} \neq-1, j=1, \ldots, r$. By Lemma 3.6 and Lemma 2.4 we can find $q \in Q^{+}$such that $q \cdot(\mathrm{kg} \cdot \xi)=\xi_{0}$. Hence $\xi=(q k g)^{-1} \cdot \xi_{0}$. 


\section{The Domain $\Xi$}

The function $\Psi_{m}$ is a holomorphic function on $\mathfrak{p}^{+} \times \mathfrak{p}^{+}$. By restricting it, we can view it as a holomorphic function on $\mathcal{D}_{b} \times \mathcal{D}_{b}$. In this section we recall the construction of the domain $\Xi^{o}$ from [6] and show that $\Xi^{o}=\left\{z \in \mathcal{D}_{b} \times \mathcal{D}_{b} \mid \Psi_{m}(z) \neq\right.$ $0\}$.

Let $\operatorname{pr}_{\mathfrak{q}}: \mathfrak{g} \rightarrow \mathfrak{q}$ be the orthogonal projection. Let $C_{G}$ be a $G$-invariant regular cone in $\mathfrak{g}$ such that $-\tau\left(C_{G}\right)=C_{G}$, and $C=C_{G} \cap \mathfrak{q}=\operatorname{pr}_{\mathfrak{q}}\left(C_{G}\right)$, cf. [5], Theorem 4.5.8. The minimal extension is the minimal cone $C_{\text {min }}$ generated by $-\operatorname{Ad}(G) Z^{0}$ and the maximal extension is the maximal cone $C_{\max }=C_{\min }^{*}$. Let $\Gamma=\Gamma\left(C_{G}\right)$ be the Olshanskii semigroup $\Gamma=G \exp i C_{G}$. Then, cf. $[5,6]$

Lemma 4.1. 1) $\Gamma \subset\left\{\gamma \in G_{\mathbb{C}} \mid \gamma^{-1} \mathcal{D}_{b} \subset \mathcal{D}_{b}\right\}$.

2) $\Gamma^{o}=G \exp i C_{G}^{o} \simeq G \times C_{G}^{o}$.

3) $\Gamma^{o} \subset\left\{\gamma \in G_{\mathbb{C}} \mid \gamma^{-1} \overline{\mathcal{D}_{b}} \subset \mathcal{D}_{b}\right\}$.

As in [6] we set $\Xi=\Gamma^{-1} \mathcal{M} \subset G_{\mathbb{C}} / H_{\mathbb{C}}$. Then $\Xi^{o}=\left(\Gamma^{o}\right)^{-1} \mathcal{M} \simeq G \times_{H}\left(-i C^{o}\right)$. In particular $\Xi^{o}$ (and $\Xi$ ) are independent of the extension $C_{G}$. The map $\Phi$ from Theorem 3.8 is defined for all $g \in G_{\mathbb{C}} / H_{\mathbb{C}}$ such that $g \cdot \xi_{0}$ exists. We have by Lemma 4.1 , part 3,

$$
\Xi^{o}=\left(\Gamma^{o}\right)^{-1} \cdot \xi_{0} \subset \mathcal{D}_{b} \times \mathcal{D}_{b} .
$$

The following has also been proven by M. Chadli, cf. [1].

Theorem 4.2. $\Xi^{o}=\left\{z \in \mathcal{D}_{b} \times \mathcal{D}_{b} \mid \Psi_{m}(z) \neq 0\right\}$. In particular $\Xi^{o}$ is open and dense in $\mathcal{D}_{b} \times \mathcal{D}_{b}$.

Proof. By Theorem 2.12 we see that the left-hand side is contained in the right hand side. Let $z=(Z, W) \in \mathcal{D}_{b} \times \mathcal{D}_{b}$ be such that $\Psi_{m}(z) \neq 0$. As $G$ acts transitively on $\mathcal{D}_{b}$ and $0 \in \mathcal{D}_{b}$, we may assume that $W=0$. Write $Z=g \cdot 0$. Then $g=k_{1} a k_{2}$, with $k_{1}, k_{2} \in K$ and $a=\exp \sum_{j=1}^{r} r_{j}\left(Y_{j}-Y_{-j}\right) \in A_{q}:=\exp \mathfrak{a}_{q}$. As $K$-stabilizes 0 we can assume that $z=(a \cdot 0,0)=\left(-i \sum_{j=1}^{r} \tanh \left(r_{j}\right) E_{j}, 0\right)$. ad $Z^{0}$ acts by multiplication by $i$ on $\mathfrak{p}^{+}$. Hence $\exp \left([\pi / 2] Z^{0}\right) \cdot\left(-i \sum_{j=1}^{r} \tanh \left(r_{j}\right) E_{j}, 0\right)=$ $\left(\sum_{j=1}^{r} \tanh \left(r_{j}\right) E_{j}, 0\right)$. Theorem 2.12, part 2, implies that $r_{j} \neq 0$ for $j=1, \ldots, r$. Let $\gamma_{1}=\exp \left(i \sum_{j=1}^{r} Y_{-j}\right) \in \Gamma$ (by Lemma 3.3). By $\mathfrak{s u}(1,1)$-reduction we get $\gamma_{1}^{-1} \cdot \xi_{o}=(E, 0)$. By Lemma 2.4 and $0<\tanh \left(r_{j}\right)<1$ we see that $\gamma_{2}:=$ $\exp \left(-\sum_{j=1}^{r} \log \left(\tanh \left(r_{j}\right)\right) H_{j}\right) \in \Gamma^{o}$. Also $\gamma_{2}^{-1} \cdot(E, 0)=\left(\sum_{j=1}^{r} \tanh \left(r_{j}\right) E_{j}, 0\right)$. The Theorem follows now as $\Gamma \Gamma^{o} \subset \Gamma^{o}$.

Example 4.3 $(\mathrm{SU}(n, n))$. Let $G=\mathrm{SU}(n, n)$. Then

$$
\mathfrak{p}^{+}=\left\{\left(\begin{array}{ll}
0 & Z \\
0 & 0
\end{array}\right) \mid Z \in M(n, \mathbb{C})\right\} \simeq M(n, \mathbb{C})
$$

and the action of $\mathrm{SU}(n, n)$ on $\mathfrak{p}^{+}$is given by

$$
\left(\begin{array}{ll}
A & B \\
C & D
\end{array}\right) \cdot Z=(A Z+B)(C Z+D)^{-1} .
$$

The maximal compact subgroup is $\mathrm{S}(\mathrm{U}(n) \times \mathrm{U}(n))$ and the bounded realization is

$$
\mathrm{SU}(n, n) / \mathrm{S}(\mathrm{U}(n) \times \mathrm{U}(n)) \simeq\left\{Z \in M(n, \mathbb{C}) \mid I_{n}-Z^{*} Z>0\right\} .
$$


The Shilov boundary is $\mathrm{U}(n)$ and $E$ corresponds to the identity matrix. The Cayley type involution $\tau$ is given as

$$
\left(\begin{array}{ll}
A & B \\
C & D
\end{array}\right) \mapsto\left(\begin{array}{cc}
D & C \\
B & A
\end{array}\right)
$$

and

$$
H=\left\{\left(\begin{array}{ll}
A & B \\
B & A
\end{array}\right) \in \mathrm{SU}(n, n)\right\} \simeq \mathrm{GL}(n, \mathbb{R})_{+} .
$$

By Lemma 2.11 we get $\Phi_{m}(Z)=\operatorname{det}(Z)^{m n}$. Thus

$$
\mathrm{SU}(n, n) / \mathrm{GL}(n, \mathbb{R})_{+} \simeq\{(Z, W) \in \mathrm{U}(n) \times \mathrm{U}(n) \mid \operatorname{det}(Z-W) \neq 0\}
$$

and $\Xi^{\circ}$ is given by

$\left\{(Z, W) \in M(n, \mathbb{C}) \times M(n, \mathbb{C}) \mid I_{n}-Z^{*} Z>0, I_{n}-W^{*} W>0, \operatorname{det}(Z-W) \neq 0\right\}$.

\section{5. $\mathbf{L}^{2}$-SPaCes And Double Coverings}

The quasi-invariant and normalized measure $\mu$ on $\mathcal{S}_{1}$ is given by

$$
\int_{\mathcal{S}_{1}} f(\xi) d \mu(\xi)=\int_{K \times K /(H \cap K \times H \cap K)} f\left(k_{1} \cdot E, k_{2} \cdot E\right) d k_{1} d k_{2} .
$$

Let $x \in G$. Define $k(x) \in K, h(x) \in H$ and $q^{-}(x) \in Q^{-}$by $x=k(x) h(x) q^{-}(x)$. Define

$$
J_{R}(x, k \cdot E)=h(x k)^{2 \rho_{+}}:=\operatorname{det}\left(\operatorname{Ad}(h) \mid \mathfrak{q}^{+}\right)=\left(\operatorname{det} \operatorname{Ad}(h) \mid \mathfrak{q}^{-}\right)^{-1} .
$$

Then by [13]

$$
\int_{\mathcal{S}_{1}} f((a \cdot Z, b \cdot W)) J_{R}(a, Z) J_{R}(b, W) d \mu(Z, W)=\int_{\mathcal{S}_{1}} f(\xi) d \mu(\xi)
$$

where $\mu$ is as in (5.1). For $g \in G_{\mathbb{C}}$ and $Z \in \mathfrak{p}^{+}$such that $g \exp Z \in P^{+} K_{\mathbb{C}} P^{-}$let $J(g, Z):=k(g, Z)^{2 \rho_{n}}$. This is the holomorphic Jacobian of the action on $\mathfrak{p}^{+}$. The connection between $J$ and $J_{R}$ is given by the following lemma.

Lemma 5.1. Suppose that $Z=k \cdot E \in \mathcal{S}$ for some $k \in K$. Then the following hold:

1) $g \cdot Z=\operatorname{Ad}(k(g k)) E$.

2) $\left|k(g, Z)^{2 \rho_{n}}\right|=h(g k)^{2 \rho_{+}}$.

Proof. Write $\mathbf{c}=\exp E k_{o} p^{-}$. Simple calculation shows that

$$
g \exp Z=k(g k) \exp E k_{o}^{-1}\left(\mathbf{c}^{-1} h(g k) \mathbf{c}\right) k_{o} k^{-1} q
$$

for some $q \in P^{-}$. The lemma now follows from $\mathbf{c}^{-1} H_{\mathbb{C}} \mathbf{c}=K_{\mathbb{C}}, \mathfrak{q}_{\mathbb{C}}^{-}=\operatorname{Ad}(\mathbf{c}) \mathfrak{p}^{-}$, and $\left|k^{2 \rho_{n}}\right|=1$ for all $k \in K$.

From Theorem 2.6 we know that $\sqrt{J(a, Z)}=k(a, Z)^{\rho_{n}}, a \in \Gamma^{-1}$ and $Z \in \operatorname{cl}\left(\mathcal{D}_{b}\right)$ is well defined if $\mathfrak{g}$ is not one of the Lie algebras $\mathfrak{s p}(2 n, \mathbb{R})$ or $\mathfrak{s o}(2,2 n+1)$. In general there is a double covering $\tilde{\Gamma}$, respectively $\tilde{G}$, of $\Gamma$, respectively $G$, such that $\sqrt{J(a, Z)}$ is defined as a function on $\tilde{\Gamma} \times \operatorname{cl}\left(\mathcal{D}_{b}\right)$ holomorphic on $\tilde{\Gamma}^{o} \times \mathcal{D}_{b}$, where $\tilde{\Gamma}^{o}=\kappa^{-1}\left(\Gamma^{o}\right), \kappa: \tilde{\Gamma} \rightarrow \Gamma$ the canonical projection. The group $\tilde{G}$ acts on $\mathcal{D}_{b}$ and $\mathcal{S}$ by $\tilde{a} \cdot Z=\kappa(a) \cdot Z$. The cocycle relation

$$
\sqrt{J(a b, Z)}=\sqrt{J(a, b \cdot Z)} \sqrt{J(b, Z)}
$$


still holds for $\tilde{\Gamma}$, cf. (1.2). By Lemma 5.1 and (5.2) we have the following theorem:

Theorem 5.2. The formula

$$
\left[\lambda_{1}(a, b) f\right](Z, W):=\sqrt{J\left(a^{-1}, Z\right)} \sqrt{J\left(b^{-1}, W\right)} f\left(a^{-1} \cdot Z, b^{-1} \cdot W\right)
$$

defines a unitary representation of $\tilde{G} \times \tilde{G}$ on $\mathbf{L}^{2}\left(\mathcal{S}_{1}\right)$.

We define also a double covering of the set $\mathcal{X}:=\left\{\xi \in \mathfrak{p}^{+} \times \mathfrak{p}^{+} \mid \Psi_{2}(\xi) \neq 0\right\}$ by

$$
\tilde{\mathcal{X}}=\left\{(\xi, z) \in \mathcal{X} \times \mathbb{C} \mid z^{2}=\Psi_{2}(\xi)\right\} .
$$

The covering projection and the square-root $\sqrt{\Psi_{2}}$ of $\Psi_{2}$ are given by the projection onto the first, respectively second, variable:

$$
p(\xi, z):=\xi \text { and } \sqrt{\Psi_{2}(\xi, z)}=z .
$$

By Theorem $2.12\left(\sqrt{J(g, Z)} \sqrt{J(g, W)} \sqrt{\Psi_{2}(Z, W)}\right)^{2}=\Psi_{2}(g \cdot(Z, W))$. Hence we have a well-defined action of $\tilde{G}$ on $\tilde{\mathcal{X}}$ given by

$$
a \cdot((Z, W), z):=(a \cdot(Z, W), \sqrt{J(a, Z)} \sqrt{J(a, W)} z) .
$$

Definition 5.3. A function $F$ in $\tilde{\mathcal{X}}$ is even, respectively odd, if $F(\xi, z)=F(\xi,-z)$ respectively $F(\xi,-z)=-F(\xi, z)$.

The pullback of the $G$-invariant (respectively quasi-invariant) measure on $\mathcal{M}$ (respectively $\mathcal{S}_{1}$ ) defines a $G$-invariant measure on $\tilde{\mathcal{M}}$ (respectively $\tilde{\mathcal{S}}_{1}$ ). We will denote this measure by the same letter as before. Thus the corresponding $\mathbf{L}^{2}$-spaces are defined. We will denote the subspace of even, respectively odd, functions by $\mathbf{L}_{\text {even }}^{2}$, respectively $\mathbf{L}_{\text {odd }}^{2}$. The same convention will be used for other function spaces. Notice that all those coverings are trivial (i.e. splits) in case $\mathfrak{g}$ is not $\mathfrak{s p}(2 n, \mathbb{R})$ or $\mathfrak{s o}(2,2 n+1)$ because we can take a global square-root of both $J$ and $\Psi_{2}$.

Lemma 5.4. Let $\kappa^{-1}(1)=\{1, \epsilon\}$. Then

$$
\sqrt{J\left(a^{-1}, Z\right)} \sqrt{J\left(b^{-1}, W\right)}=\sqrt{J\left(a^{-1} \epsilon, Z\right)} \sqrt{J\left(b^{-1} \epsilon, W\right)} .
$$

Proof. Let $\epsilon$ be as in the lemma. Then $\sqrt{J(\epsilon, Z)}=-1$ for every $Z$. The claim follows now from (5.3).

It follows that the representation $\lambda_{1}$ factors to $\tilde{G} \times \tilde{G} /\{(1,1),(\epsilon, \epsilon)\}$. Restricted to the diagonal $\Delta(\tilde{G})$ it defines a representation of $\Delta(\tilde{G}) /\{(1,1),(\epsilon, \epsilon)\} \simeq G$. We notice also that Lemma 5.4 and Theorem 2.12 imply that action of $\tilde{G}$ and $\tilde{\Gamma}$ on $\tilde{\Xi}$ factors to an action of $G$ and $\Gamma$ as $\epsilon$ acts trivially. The same argument shows that the covering $\tilde{\Xi} \rightarrow \Xi$ is trivial on every $G$ and $\Gamma^{o}$-orbit.

Lemma 5.5. 1) Let $p^{-1}\left(\xi_{o}\right)=\left\{\tilde{\xi}_{o}, \tilde{\xi}_{1}\right\}$. Let $\mathcal{M}_{j}:=G \cdot \tilde{\xi}_{j}, j=0,1$. Then $\tilde{\mathcal{M}}=\mathcal{M}_{o} \cup \mathcal{M}_{1}$ (disjoint union) and both $\mathcal{M}_{o}$ and $\mathcal{M}_{1}$ are isomorphic to $\mathcal{M}$ as $G$-spaces.

2) The spaces $\mathbf{L}^{2}(\tilde{\mathcal{M}})_{\text {even }}$ and $\mathbf{L}^{2}(\tilde{\mathcal{M}})_{\text {odd }}$ are $G$-invariant subspaces both of which are equivalent to $\mathbf{L}^{2}(\mathcal{M})$. The same holds also for the spaces $\mathbf{L}^{2}\left(\mathcal{M}_{o}\right)$ and $\mathbf{L}^{2}\left(\mathcal{M}_{1}\right)$. We have

$$
\mathbf{L}^{2}(\tilde{\mathcal{M}}) \simeq \mathbf{L}^{2}(\tilde{\mathcal{M}})_{\text {even }} \oplus \mathbf{L}^{2}(\tilde{\mathcal{M}})_{\text {odd }} \simeq \mathbf{L}^{2}\left(\mathcal{M}_{o}\right) \oplus \mathbf{L}^{2}\left(\mathcal{M}_{1}\right) .
$$


Proof. 1) Let $(m, z) \in p^{-1}(\mathcal{M})$. Let $m=g \cdot \mathbf{o}$. As $z$ is a square-root of $\Psi_{2}(m)$ we have also fixed a square root $z_{o}$ of $\Psi_{2}(\mathbf{o})$. It follows that $(m, z)=g \cdot\left(\mathbf{o}, z_{o}\right)$. That $\mathcal{M}_{0} \simeq \mathcal{M}_{1} \simeq \mathcal{M}$ is obvious as the stabilizer of $\xi_{o}$ and $\xi_{1}$ is in both cases $H$.

2) If $f \in \mathbf{L}^{2}(\tilde{\mathcal{M}})$ is continuous, then we can write

$$
f(m, z)=\frac{1}{2}(f(m, z)+f(m,-z))+\frac{1}{2}(f(m, z)-f(m,-z))
$$

which defines a continuous projection onto the space of even, respectively odd, functions. On the other hand, if $f$ is even or odd, then $f$ is determined by the restriction to $\mathcal{M}_{o}$ or $\mathcal{M}_{1}$. This gives the needed isomorphism.

We prove the following lemma in the same way as part 1) in the last lemma:

Lemma 5.6. Let $\Xi_{i}^{o}:=\left(\Gamma^{o}\right)^{-1} \cdot \mathcal{M}_{i}$. Then $\tilde{\Xi}^{o}=\Xi_{o}^{o} \cup \Xi_{1}^{o}$.

The following theorem follows now by the definition of $\Psi_{1}$ and $\Psi_{2}$, cf. (2.1).

Theorem 5.7. Let $\lambda$ be the regular representation of $G$ on $\mathbf{L}^{2}(\mathcal{M})$. Then the following hold:

1) The $G$-invariant measure on $\Phi(\mathcal{M}) \subset \mathcal{S}_{1}$ is up to a constant given by

$$
\int_{\mathcal{S}_{1}} f(\xi)\left|\Psi_{2}(\xi)\right|^{-1} d \mu(\xi) .
$$

2) The map $\mathbf{L}^{2}\left(\mathcal{S}_{1}\right) \ni f \mapsto\left(f\left|\Psi_{2}\right|^{1 / 2}\right) \mid \mathcal{M} \in \mathbf{L}^{2}(\mathcal{M})$ is a G-equivariant isometry of $\left(\mathbf{L}^{2}\left(\mathcal{S}_{1}\right), \lambda_{0} \mid G\right)$ onto $\left(\mathbf{L}^{2}(\mathcal{M}), \lambda\right)$.

3) If $\mathfrak{g} \neq \mathfrak{s p}(2 n, \mathbb{R})$ or $\mathfrak{g} \neq \mathfrak{s o}(2,2 n+1)$, then

$$
\mathbf{L}^{2}\left(\mathcal{S}_{1}\right) \ni f \mapsto\left(f \Psi_{1}\right) \mid \mathcal{M} \in \mathbf{L}^{2}(\mathcal{M})
$$

defines a $G$-equivariant isometry $\left(\mathbf{L}^{2}\left(\mathcal{S}_{1}\right), \lambda_{1} \mid G\right)$ onto $\left(\mathbf{L}^{2}(\mathcal{M}), \lambda\right)$.

4) Let $f \in \mathbf{L}^{2}\left(\mathcal{S}_{1}\right)$. Then $(f \circ p) \sqrt{\Psi_{2}} \in \mathbf{L}^{2}(\tilde{\mathcal{M}})_{\text {odd }}$ and

$$
\mathbf{L}^{2}\left(\mathcal{S}_{1}\right) \ni f \mapsto(f \circ p) \sqrt{\Psi_{2}} \in \mathbf{L}^{2}(\tilde{\mathcal{M}})_{\text {odd }}
$$

is an isometric G-isomorphism.

Remark 5.8. The above theorem shows in particular that, restricted to the diagonal, the different representations of $G=\Delta(G)$ on $\mathbf{L}^{2}\left(\mathcal{S}_{1}\right)$, i.e. those defined by using the real Jacobian, respectively the complex Jacobian, are equivalent.

\section{The Hardy Spaces}

For a complex manifold $U$ let $\mathcal{O}(U)$ be the space of holomorphic functions on $U$. For $\gamma \in \Gamma$ and $f \in \mathcal{O}\left(\Xi^{o}\right)$ let $\gamma \cdot f(\xi)=f\left(\gamma^{-1} \xi\right)$. The Hardy space $\mathcal{H}_{2}$ is the space of holomorphic functions on $\Xi^{o}$ such that

$$
\sup _{\gamma \in \Gamma^{o}}\|(\gamma \cdot f) \mid \mathcal{M}\|_{\mathbf{L}^{2}(\mathcal{M})}<\infty,
$$

cf. [6]. If $f \in \mathcal{H}_{2}$ the $\mathbf{L}^{2}$-limit $\beta(f):=\lim _{\gamma \rightarrow 1}(\gamma \cdot f) \mid \mathcal{M}$ exists and defines a $G$ equivariant isometry into $\mathbf{L}^{2}(\mathcal{M})$. The image of $\beta$ is given by the direct sum of the holomorphic discrete series constructed in $[17,18]$. Let $\pi$ be an irreducible unitary representation of $K$ on the finite dimensional Hilbert space $\mathbf{V}_{\pi}$. Denote by the same letter $\pi$ the holomorphic extension to $K_{\mathbb{C}}$. Let $\pi^{*}$ denote the contragradient representation on $\mathbf{V}_{\pi}^{*}$. We will assume that $\pi^{*}$ contains a non-zero $H_{\mathbb{C}} \cap K_{\mathbb{C}}$ fixed vector $u_{o}$. We normalize $u_{o}$ to have norm 1 . Let $\mathfrak{t}$ be a Cartan subalgebra of $\mathfrak{k}$ and $\mathfrak{g}$ containing $\mathfrak{a}$. Let $\mu_{\pi} \in i \mathfrak{t}^{*}$ be the highest weight of $\pi$ with respect to the positive 
system $\Delta_{k}^{+}$, cf. Lemma 2.3. Then $\mu_{\pi} \in i \mathfrak{a}^{*}$. The set $H_{\mathbb{C}} K_{\mathbb{C}} P^{+}$is open and dense in $G_{\mathbb{C}}$. For $x^{-1}=h\left(x^{-1}\right) k_{H}\left(x^{-1}\right) p^{+}\left(x^{-1}\right) \in H_{\mathbb{C}} K_{\mathbb{C}} P^{+}$define

$$
\Phi_{\pi, u}(x):=\left\langle u, \pi^{*}\left(k_{H}\left(x^{-1}\right)^{-1}\right) u_{o}\right\rangle, \quad u \in \mathbf{V}_{\pi}
$$

Let

$$
\rho=\frac{1}{2} \sum_{\alpha \in \Delta^{+}} m_{\alpha} \alpha=\frac{1}{2} \sum_{j=1}^{r}(1+d(r-j)) \gamma_{j}
$$

where $m_{\alpha}=\operatorname{dim} \mathfrak{g}_{\alpha}$. Then the following holds, cf. [6]:

Theorem 6.1. Let $\mathcal{E}:=\left\{\mu \in \mathfrak{t}_{\mathbb{C}}^{*} \mid \forall \alpha \in \Delta_{n}^{+}: \Re\langle\mu+\rho, \alpha\rangle<0\right\}$. Then we have

1) $\Phi_{\pi, u} \in \mathcal{O}\left(P^{+} K_{\mathbb{C}} H_{\mathbb{C}}\right)$.

2) $\forall p \in P^{+}, \forall h \in H_{\mathbb{C}}$, and for all $x \in P^{+} K_{\mathbb{C}} H_{\mathbb{C}}$ we have $\Phi_{\pi, u}(p x h)=\Phi_{\pi, u}(x)$. In particular $\Phi_{\pi, u}$ can be viewed as a $P^{+}$-invariant holomorphic function on the open dense domain $P^{+} K_{\mathbb{C}} \cdot \mathbf{o} \subset G_{\mathbb{C}} / H_{\mathbb{C}}$.

3) $\Xi \subset P^{+} K_{\mathbb{C}} \cdot \mathbf{o} \subset G_{\mathbb{C}} / H_{\mathbb{C}}$. Thus by restriction $\Phi_{\pi, u} \in \mathcal{O}\left(\Xi^{o}\right)$.

4) $\forall k \in K_{\mathbb{C}}, \forall x \in P^{+} K_{\mathbb{C}} \cdot \mathbf{o}$ we have

$$
\Phi_{\pi, u}\left(k^{-1} x\right)=\Phi_{\pi, \pi(k) u}(x) .
$$

5) If $u \neq 0$, then $\Phi_{\pi, u} \mid \mathcal{M} \in \mathbf{L}^{2}(\mathcal{M})$ if and only if $\mu_{\pi} \in \mathcal{E}$. In that case $\Phi_{\pi, u}$ generates an irreducible $G$-invariant subspace $\mathbf{E}_{\pi}$ in $\mathbf{L}^{2}(\mathcal{M})$. Furthermore $\mathbf{V}_{\pi} \ni u \mapsto \Phi_{\pi, u} \in \mathbf{E}_{\pi}$ is a $K$-intertwining operator.

6) If $u \neq 0$, then $\Phi_{\pi, u} \in \mathcal{H}_{2}$ if and only if $\mu_{\pi} \in \mathcal{E}$. In that case $\beta\left(\Phi_{\pi, u}\right)=$ $\Phi_{\pi, u} \mid \mathcal{M}$.

7) $\operatorname{Im} \beta=\bigoplus_{\mu_{\pi} \in \mathcal{E}} \mathbf{E}_{\pi}$.

Lemma 6.2. Let $u$ be a weight vector of weight $\mu$. Then

$$
\Phi_{\pi, u}(E, 0)=\left(\prod_{j=1}^{r} 2^{\mu\left(H_{j}\right) / 2}\right)\left\langle u, u_{o}\right\rangle
$$

In particular $\Phi_{\pi, u}(E, 0)=0$ if $\mu \notin i \mathfrak{a}^{*}$.

Proof. We recall that $\exp -i \sum_{j=1}^{r} Y_{-j} \cdot \xi=(E, 0)$. On the other hand $\mathfrak{s u}(1,1)$ reduction shows that

$$
k_{H}\left(\exp \sum_{j=1}^{r} Y_{-j}\right)=\exp \frac{1}{2} \sum_{j=1}^{r}(\log 2) H_{j}
$$

The claim follows now from Theorem 6.1, part 4 .

Theorem 6.3. Suppose that $Z-W=\operatorname{Ad}(k) \sum_{j=1}^{r} x_{j} E_{j} \in \operatorname{Ad}(K)\left\{\sum_{j=1}^{r} x_{j} E_{j} \mid\right.$ $\left.\forall j: x_{j} \neq 0\right\}$ be such that $\Phi_{\pi, u}(Z, W)$ is defined. Choose $r_{j} \in \mathbb{C}$ such that $x_{j}=$ $\exp 2 r_{j}$. Write $\pi\left(k^{-1}\right) u=\sum_{\nu} a_{\nu}(k) u_{\nu}$, where $u_{\nu}$ are weight vectors of weight $\mu_{\mu}$. Then

$$
\Phi_{\pi, u}(Z, W)=\sum_{\nu} a_{\nu}(k)\left(\prod_{j=1}^{r} x_{j}^{-\mu_{\nu}\left(H_{j}\right) / 2}\right)\left(\prod_{j=1}^{r} 2^{\mu_{\nu}\left(H_{j}\right) / 2}\right)\left\langle u_{\nu}, u_{o}\right\rangle .
$$


Proof. Let $\Phi_{\nu}:=\Phi_{\pi, u_{\nu}}$. By Theorem 6.1 we know that $\Phi_{\pi, u}$ is $P^{+}$-invariant. As $\exp \sum r_{j} H_{j} \cdot(E, 0)=\left(\sum x_{j} E_{j}, 0\right)$ we get

$$
\begin{aligned}
\Phi_{\pi, u}(Z, W) & =\Phi_{\pi, u}(Z-W, 0) \\
& =\sum_{\nu} a_{\nu}(k) \Phi_{\nu}\left(\sum_{j=1}^{r} x_{j} E_{j}, 0\right) \\
& =\sum_{\nu} a_{\nu}(k) \Phi_{\nu}\left(\exp \left(\sum_{j=1}^{r} r_{j} H_{j}\right) \cdot(E, 0)\right) \\
& =\sum_{\nu} a_{\nu}(k) \Phi_{\pi, \exp \left(-\sum_{j=1}^{r} r_{j} H_{j}\right) u_{\nu}}(E, 0) \\
& =\sum_{\nu} a_{\nu}(k) \exp \left(-\sum_{j=1}^{r} r_{j} \mu_{\nu}\left(H_{j}\right)\right) \Phi_{\nu}(E, 0) \\
& =\sum_{\nu} a_{\nu}(k)\left(\prod_{j=1}^{r} x_{j}^{-\mu_{\nu}\left(H_{j}\right) / 2}\right) \Phi_{\nu}(E, 0) \\
& =\sum_{\nu} a_{\nu}(k)\left(\prod_{j=1}^{r} x_{j}^{-\mu_{\nu}\left(H_{j}\right) / 2}\right)\left(\prod_{j=1}^{r} 2^{\mu_{\nu}\left(H_{j}\right) / 2}\right)\left\langle u_{\nu}, u_{o}\right\rangle .
\end{aligned}
$$

From this the theorem now follows.

Remark 6.4. Notice that the above theorem applies to $\Phi_{\pi, u} \mid \Xi$.

We want now to relate the Hardy space $\mathcal{H}_{2}$ to the classical Hardy space. The classical Hardy space $\mathcal{H}^{c l}$ is the space of holomorphic functions on $\mathcal{D}_{b} \times \mathcal{D}_{b}$ that extend to $\mathbf{L}^{2}$-functions on $\mathcal{S}_{1}$. We have a representation of $\tilde{G}$ on the Hardy space given by

$$
\left[\lambda_{H s p}(a) f\right]=\sqrt{J\left(a^{-1}, Z\right)} f\left(a^{-1} \cdot Z\right) .
$$

In particular the boundary value map is $G$-equivariant, resp. $\tilde{G}$-equivariant. We remark that we could have replaced, in all the constructions $G, \Gamma$ and $\Xi^{o}$ by the corresponding coverings to get the corresponding Hardy space $\tilde{\mathcal{H}}_{2}$ and boundary value map $\tilde{\beta}: \tilde{\mathcal{H}}_{2} \rightarrow \Lambda^{2}(\tilde{\mathcal{M}})$. Note that it is known from [6] and [17] that the image of $\tilde{\beta}$ is again the sum over the holomorphic discrete series constructed in the same way as before, using the lifting of the map $k_{H}$. $\tilde{\mathcal{H}}_{2}$ is a $G$-space as the action of $\epsilon$ on $\tilde{\mathcal{X}}$ is trivial and $\beta$ is an isometric $G$-map. The boundary value map maps even functions into even functions and odd functions into odd functions. Then Lemma 5.5 and Lemma 5.6 show that

Lemma 6.5. We have the decomposition of $\tilde{\mathcal{H}}_{2}$ into equivalent $G$-spaces

$$
\tilde{\mathcal{H}}_{2} \simeq \tilde{\mathcal{H}}_{2 \text { even }} \oplus \tilde{\mathcal{H}}_{2 \text { odd }}
$$

and both $\tilde{\mathcal{H}}_{2 \text { even }}$ and $\tilde{\mathcal{H}}_{2 \text { odd }}$ are equivalent to $\mathcal{H}_{2}$.

Theorem 6.6. Assume that $\mathfrak{g} \neq \mathfrak{s p}(2 n, \mathbb{R})$ or $\mathfrak{g} \neq \mathfrak{s o}(2,2 n+1)$. Define $\mathbf{I}: \mathcal{H}^{c l} \rightarrow$ $\mathcal{H}_{2}$ by $\mathbf{I}(f):=\left(f \Psi_{1}\right) \mid \Xi^{\circ}$. Then $\mathbf{I}$ is a $G$-equivariant unitary $G$-isomorphism. 
Proof. $\mathbf{I}$ is injective as $\Xi^{o}$ is open and dense in $\mathcal{D}_{b} \times \mathcal{D}_{b}$ and $\Psi_{1}$ is non-zero on $\Xi^{o}$.

$$
\begin{aligned}
\mathbf{I}(f)\left(g^{-1} \cdot(Z, W)\right) & =f\left(g^{-1} \cdot(Z, W)\right) \Psi_{1}\left(g^{-1} \cdot(Z, W)\right) \\
& =\sqrt{J\left(g^{-1}, Z\right)} \sqrt{J\left(g^{-1}, W\right)} f\left(g^{-1} \cdot(Z, W)\right) \Psi_{1}(Z, W) \\
& =I(\lambda(g) f)(Z, W) .
\end{aligned}
$$

So what we have to show is that for $f \in \mathcal{H}_{2}$ the function $f / \Psi_{1}$ is bounded. It follows then that this function extends to a holomorphic function on $\mathcal{D}_{b} \times \mathcal{D}_{b}$ and is actually continuous on $\mathcal{S}_{1}$. We will prove this in the following steps.

By Theorem 2.12 we have

$$
\Psi_{1}(Z, W)=\Psi_{1}(Z-W, 0)=k^{2 \rho_{n}} \prod_{j=1}^{r} x_{j}^{1+\frac{d(r-1)}{2}}
$$

where $Z-W=\operatorname{Ad}(k) \sum_{j=1}^{r} x_{j} E_{j}$. Choose $r_{j} \in \mathbb{C}$ such that $e^{2 r_{j}}=x_{j}$. This is possible as $x_{j} \neq 0$. Let $u \in \mathbf{V}_{\pi}$ and write $k^{-1} u=\sum a_{\nu}(k) u_{\nu}$, where $a_{\nu}$ is a continuous function of $k$ and $u_{\nu}$ is a weight vector for the weight $\mu_{\nu}$. Then by Theorem 6.3 we now get

$$
\frac{\Phi_{\pi, u}(Z, W)}{\Psi_{1}(Z, W)}=\sum_{\nu}\left(k^{-2 \rho_{n}} a_{\nu}(k)\left\langle u_{\nu}, u_{o}\right\rangle \prod_{j=1}^{r} 2^{\mu_{n}\left(H_{j}\right) / 2}\right) \prod_{j=1}^{r} x_{j}^{-\left(\frac{\mu_{\nu}\left(H_{j}\right)}{2}+1+\frac{d(r-1)}{2}\right)} .
$$

We will now show in Lemma 6.8 that actually $-\left(\frac{\mu_{\nu}\left(H_{j}\right)}{2}+1+\frac{d(r-1)}{2}\right) \geq 0$. That will then prove the theorem.

Lemma 6.7. Let $\mu=\mu_{\pi}$ be the highest weight of $\pi \in \hat{K}$ such that $\pi$ has a $K \cap H$ fixed vector and $\langle\mu+\rho, \alpha\rangle<0$ for every $\alpha \in \Delta_{n}^{+}$. Then $\frac{\left\langle\mu, H_{j}\right\rangle}{2}+1+\frac{d(r-1)}{2} \leq 0$.

Proof. Write $\mu=\sum_{j=1}^{r} n_{j} \gamma_{j}$. As $\mu$ is $\Delta_{k}^{+}$-dominant, $n_{1} \geq n_{2} \geq \cdots \geq n_{r}$. By [19] we have $n_{j} \in \mathbb{Z}$ for every $j$. As $\rho=\frac{1}{2} \sum_{j=1}^{r}(1+d(r-j)) \gamma_{j}$ and $H_{j}$ corresponds to $2 \gamma_{j} /\left|\gamma_{j}\right|$ under the isomorphism $i \mathfrak{a} \simeq i \mathfrak{a}^{*}$, the condition $\langle\mu+\rho, \alpha\rangle<0$ reads

$$
\forall j: 2 n_{j}+1+d(r-j)<0 .
$$

On the other hand $\rho_{n}=\frac{1}{2}\left(1+\frac{d(r-1)}{2}\right) \sum_{j=1}^{r} \gamma_{j}$. Hence by $(6.2)$

$$
\begin{aligned}
\frac{\left\langle\mu, H_{j}\right\rangle}{2}+1+\frac{d(r-1)}{2} & \leq n_{1}+1+\frac{d(r-1)}{2} \\
& =\frac{1}{2}\left(2 n_{1}+1+d(r-1)\right)+\frac{1}{2} \\
& <\frac{1}{2} .
\end{aligned}
$$

But $n_{j}, \frac{d(r-j)}{2} \in \mathbb{Z}$. Hence $n_{j}+1+\frac{d(r-j)}{2} \leq 0$ and the claim follows for the roots $\gamma_{j}$. But the other positive noncompact roots are of the form $\frac{1}{2}\left(\gamma_{i}+\gamma_{j}\right)$ and the claim follows.

Lemma 6.8. Let $\pi$ be as above. Let $\mu$ be a weight of $\pi$ such that $\mu \in i \mathfrak{a}^{*}$. Then $\frac{\left\langle\mu, H_{j}\right\rangle}{2}+1+\frac{d(r-1)}{2} \leq 0$ for every $j$. 
Proof. If $\mu$ is a weight, then $\mu_{\pi}-\frac{1}{2} \sum_{i<j} n_{i j}\left(\gamma_{i}-\gamma_{j}\right)$ with $n_{i j} \geq 0$. But then

$$
\begin{aligned}
\frac{\left\langle\mu, H_{1}\right\rangle}{2}+1+\frac{d(r-1)}{2} & =\frac{\left\langle\mu_{\pi}, H_{1}\right\rangle}{2}+1+\frac{d(r-1)}{2}-\frac{1}{2} \sum_{1<j} n_{1 j} \\
& \leq \frac{\left\langle\mu_{\pi}, H_{1}\right\rangle}{2}+1+\frac{d(r-1)}{2} \\
& \leq 0 .
\end{aligned}
$$

Let $s_{i j}=s_{\frac{1}{2}\left(\gamma_{i}-\gamma_{j}\right)}$. Then $s_{i j}$ maps the set of weights occurring in the lemma into itself. Furthermore $H_{j}=s_{\frac{1}{2}\left(\gamma_{1}-\gamma_{j}\right)}\left(H_{1}\right)$. Therefore

$$
\begin{aligned}
\frac{\left\langle\mu, H_{j}\right\rangle}{2}+1+\frac{d(r-1)}{2} & =\frac{\left\langle\mu, s_{1, j}\left(H_{1}\right)\right\rangle}{2}+1+\frac{d(r-1)}{2} \\
& =\frac{\left\langle s_{1, j}(\mu), H_{1}\right\rangle}{2}+1+\frac{d(r-1)}{2} \leq 0 .
\end{aligned}
$$

From this the lemma follows.

For the general case we notice that if $f \in \mathcal{H}^{c l}$, then $(f \circ p) \sqrt{\Psi_{2}} \in \tilde{\mathcal{H}}_{2}$. Theorem 6.9 follows in the same manner using Lemma 6.5.

Theorem 6.9. Define $\tilde{\mathbf{I}}: \mathcal{H}^{c l} \rightarrow \tilde{\mathcal{H}}_{2 o d d}$ by

$$
\tilde{\mathbf{I}}(f):=(f \circ p) \sqrt{\Psi_{2}} .
$$

Then $\tilde{\mathbf{I}}$ is a G-equivariant unitary G-isomorphism.

We end the paper by indicating how the explicit results above allow us to calculate the Cauchy-Szegö kernel for the Hardy space on $\mathcal{M}$. As a special case we obtain the $z=1$ specialized version of Heine's formula, as mentioned in the introduction. Indeed, by the reduction technique via the strongly orthogonal roots, practically all information is contained in the $\mathrm{SU}(1,1)$ case. Thus recall Lemma 2.7. We here have the explicit isomorphism

$$
\mathbf{I}: F(z, w) \rightarrow f(z, w)=(z-w) F(z, w)
$$

from the classical Hardy space to that of $\mathcal{M}=G / H$, the hyperboloid of one sheet realized as the off-diagonal subset of $\mathcal{S} \times \mathcal{S}$, with $\mathcal{S}$ the unit circle. Here $z, w$ are complex numbers of modulus less than one, $F(z, w)$ is in the tensor product of the Hardy space for $\mathcal{S}$ with itself, and $f(z, w)$ is the corresponding function (on the off-diagonal) in the Hardy space for $\mathcal{M}$. Since the Cauchy-Szegö kernel is (up to a constant)

$$
K_{c l}\left((z, w),\left(z_{1}, w_{1}\right)\right)=\frac{1}{1-z \overline{z_{1}}} \frac{1}{1-w \overline{w_{1}}}
$$

for the classical space, we find in these coordinates the Cauchy-Szegö kernel for $\mathcal{M}$ as

$$
K\left((z, w),\left(z_{1}, w_{1}\right)\right)=\frac{z-w}{1-z \overline{z_{1}}} \frac{\overline{z_{1}}-\overline{w_{1}}}{1-w \overline{w_{1}}}
$$

by using the above isomorphism in both the holomorphic and the anti-holomorphic coordinates. We may as usual just set one variable equal to the base point, i.e. set $z_{1}=-w_{1}=1$; since it suffices to know the kernel in the direction of the Cartan subspace, we consider the diagonal action of the one-parameter group

$$
g=\left(\begin{array}{cc}
\cosh t & i \sinh t \\
-i \sinh t & \cosh t
\end{array}\right)
$$


on the base point $(1,-1)$ in $\mathcal{S} \times \mathcal{S}$. This gives us

$$
z=g \cdot 1=\frac{u}{\bar{u}}, w=g \cdot(-1)=\frac{-\bar{u}}{u}
$$

where $u=\cosh t+i \sinh t$. This we may insert in the above expression for $K$ to obtain

$$
K=2 \frac{u^{2}+\bar{u}^{2}}{(\bar{u}-u)(u-\bar{u})}=\frac{1}{(\sinh t)^{2}}
$$

which may also be written as

$$
K=\frac{2}{\cosh 2 t-1} .
$$

In these same coordinates the invariant distribution for the holomorphic discrete series is $2(2 n+1) Q_{n}(\cosh 2 t)$, so since the Cauchy-Szegö kernel is the sum of these, we get Heine's formula.

Observe that the general situation allows the calculation of the Cauchy-Szegö kernel as a product of the formulas for $\mathrm{SU}(1,1)$ : Again it suffices to let the antiholomorphic argument be the base point and the holomorphic argument along the direction of the Cartan subspace. Since the classical Hardy space for $\mathcal{D}_{b}$ has the kernel

$$
K_{c l}\left(z, z_{1}\right)=\prod_{j=1}^{r}\left(1-z_{j} \overline{z_{1, j}}\right)^{-(1+d(r-1) / 2)}
$$

and the classical kernel for the tensor product, i.e. the classical Hardy space we are comparing with, is the product of two such terms as in the rank-one case, we obtain the following formula for the Cauchy-Szegö kernel for the general Cayley type space:

$$
K=\prod_{j=1}^{r}\left(\cosh 2 t_{j}-1\right)^{-(1+d(r-1) / 2)} .
$$

Here the coordinates $t_{j}$ are the same as in the $\mathrm{SU}(1,1)$ case, one for each strongly orthogonal root. It would be interesting to calculate the exact normalizations between the generalized Legendre functions in several variables $Q_{\lambda}$ and the invariant distributions for the holomorphic discrete series to make explicit the formula

$$
K=\sum_{\lambda} Q_{\lambda}
$$

where now the left-hand side is known by the above.

\section{REFERENCES}

1. M. Chadli, Domaine complexe assocé à un espace symétrique de type Cayley. C.R. Acad. Sci. Paris 321 (1995), 1157-1161. MR 97f:22022

2. Harish-Chandra, Representations of semi-simple Lie groups. IV. Amer. J. Math. 77 (1955), 743-777. MR 17:282c

3. S. Helgason, Differential Geometry, Lie Groups and Symmetric Spaces. Academic Press. New York/London, 1978. MR 80k:53081

4. S. Helgason, Groups and geometric analysis. Integral geometry, invariant differential operators and spherical functions. Academic Press. New York/London, 1984. MR 86c:22017

5. J. Hilgert, G. Ólafsson, Causal Symmetric Spaces, Geometry and Harmonic Analysis. Perspectives in Mathematics 18, Academic Press, 1996. MR 97m:43006 
6. J. Hilgert, G. Ólafsson, B. Ørsted, Hardy spaces on affine symmetric spaces. J. reine und angew. Math. 415 (1991), 189-218. MR 92h:22030

7. S. Kaneyuki, On orbit structure of compactifications of parahermitian symmetric spaces. Japan. J. Math. 13 (1987), 333-370. MR 88m:53094

8. A. Korányi, J. A Wolf, Realization of Hermitian symmetric spaces as generalized half-planes. Ann. of Math. 81 (1965), 265-288. MR 30:4980

9. K. Koufany, Réalisation des espaces symétriques de type Cayley. C. R. Acad. Sci. Paris. 318 (1994), 425-428. MR 95h:32036

10. K. Koufany, B. Ørsted, Espace de Hardy sur le semi-groupe métaplectique. C. R. Acad. Sci. Paris, to appear. MR 96m:22017

11. O. Loos, Symmetric Spaces, I: General Theory. W.A. Benjamin, Inc., New York, 1969. MR 39:365a

12. V. F. Molchanov, Holomorphic discrete series for hyperboloids of Hermitian type, J. Funct. Anal. 147 (1997), 26-50. CMP 97:13

13. G. Ólafsson, Fourier and Poisson transformation associated to a semisimple symmetric space. Invent. math. 90 (1987), 605-629. MR 89d:43011

14. G. Ólafsson, Causal symmetric spaces. Mathematica Gottingensis. 15 (1990).

15. G. Ólafsson, Symmetric spaces of Hermitian type. Diff. Geom. and Appl. 1 (1991), 195-233. MR 94g:22034

16. G. Ólafsson, Spherical Functions and Spherical Laplace Transform on Ordered Symmetric Spaces. Preprint, 1997.

17. G. Ólafsson, B. Ørsted, The holomorphic discrete series for affine symmetric spaces, I. J. Funct. Anal. 81 (1988), 126-159. MR 89m:22021

18. G. Ólafsson, B. Ørsted, B. The holomorphic discrete series of an affine symmetric space and representations with reproducing kernels. Trans. Amer. Math. Soc. 326 (1991), 385-405. MR 91j:22014

19. W. Schmid, Die Randwerte holomorpher Funktionen auf hermitesch symmetrischen Räumen. Invent. Math. 9 (1969), 61-80. MR 41:3806

20. R. T. Stanton, Analytic extension of the holomorphic discrete series. Amer. J. of Math. 108 (1986), 1411-1424. MR 88b:22013

21. E. T. Whittaker, G. N. Watson, Modern Analysis. Cambridge 1946. MR 97k:01072

Department of Mathematics, Louisiana State University, Baton Rouge, Louisiana 70803

E-mail address: olafsson@math.1su.edu

Matematisk Institut, Odense Universitet, Campusvej 55, DK-5230 Odense M, Denmark

E-mail address: orsted@imada.ou.dk 